Abel S. Vieira, Rodney A. Stewart, Roberto Lamberts, Cara D. Beal, Residential solar water heaters in Brisbane, Australia: Key performance parameters and indicators, Renewable Energy, 2017, ISSN 0960-1481, https://doi.org/10.1016/j.renene.2017.09.054.

(http://www.sciencedirect.com/science/article/pii/S0960148117309163)

\title{
Residential solar water heaters in Brisbane, Australia: key performance parameters and indicators
}

Abel S. Vieira ${ }^{\mathrm{a}}$, Rodney A. Stewart ${ }^{\mathrm{a}}$, Roberto Lamberts ${ }^{\mathrm{b}}$, Cara D. Beal ${ }^{\mathrm{c}}$

${ }^{a}$ School of Engineering, Griffith University, Gold Coast Campus, Queensland 4222, Australia; ${ }^{b}$ Federal University of Santa Catarina, Department of Civil Engineering, Field of Energy Efficiency in Buildings, Florianópolis, 88040-900, SC, Brazil; ' ${ }^{\mathrm{c}}$ Smart Water Research Centre and School of Engineering, Griffith University, Gold Coast Campus, Queensland 4222, Australia.

\section{Corresponding author:}

Professor Rodney Stewart

School of Engineering, Gold Coast Campus, Griffith University, Parklands Drive, Southport, QLD 4222 Australia;

Phone: $\quad$ +6175552 8778

Email: r.stewart@griffith.edu.au

\section{Highlights:}

- Performance frameworks must consider site and user behaviour parameters.

- Policies on SWHs need to provide directions on best practices for operation.

- Dust accumulation on collectors impacts significantly SWHs performance.

- Split SWHs generally outperform thermosiphon SWHs.

- Efficient solar collectors improve the protection against Legionella spp.

\section{Abstract}

A multi-parametric sensitivity analysis of Solar Water Heater (SWH) systems was undertaken for the city of Brisbane in Australia using computational models calibrated by experimental data. The models were calculated using EnergyPlus 8.6. The following technical specification parameters were assessed in the modelling: (i) solar collector efficiency; (ii) solar collector area; (iii) tank volume; (iv) tank heat loss; (v) electric back-up heating power rate; (vi) electric back-up heating position (height) for vertical tanks; and (vii) electric back-up heating temperature range. The site-specific parameters included: (i) solar collector direction; (ii) solar collector tilt angle; (iii) solar collector shadowing; (iv) solar collector dust accumulation; (v) hot water pipe insulation; (vi) hot water pipe length; (vii) electricity tariff time-of-use; and (viii) cold water temperature. User behaviour patterns were comprised of the following parameters: (i) end-use water temperature; (ii) end-use water demand; and (iii) end-use time-of-use. For all parameters, two system types were assessed, namely: (i) thermosiphon systems with natural (passive) circulation in collectors and unstratified horizontal hot water storage tanks; and (ii) split systems with forced (pumped) circulation in collectors and stratified vertical hot water storage tanks. The performance of SWHs was analysed considering both energy performance indicators (i.e. total and peak-hour energy consumption, solar fraction and energy intensity) and level of service indicators (i.e. compliance with recommended hot water temperatures for Legionella spp. control and comfort levels). Notwithstanding the prevalence of thermosiphon systems among SWH technologies, results indicate that split systems usually outperformed thermosiphon systems both in terms of energy efficiency and level of service, and hence should be a preferred option for energy efficiency initiatives and policies.

Keywords: solar water heater; sensitivity analysis; site-specific variables; technical specifications; energy performance; level of service. 


\section{Nomenclature}

$\begin{array}{ll}\text { CV } & \text { Coefficient of variation } \\ \text { CWT } & \text { Cold water temperature } \\ \text { EHP } & \text { Electric back-up heating position } \\ \text { EHT } & \text { Electric back-up heating temperature range } \\ \text { EPR } & \text { Electric back-up heating power rate } \\ \text { ETD } & \text { Electricity tariff time-of-use } \\ \text { EWD } & \text { End-use water demand } \\ \text { EWT } & \text { End-use water temperature } \\ \text { EWU } & \text { End-use water time-of-use } \\ \text { GHG } & \text { Greenhouse Gas } \\ \text { HPI } & \text { Hot water pipe insulation } \\ \text { HPL } & \text { Hot water pipe length } \\ \text { IoT } & \text { Internet of Things } \\ \text { LE } & \text { Lower-extreme energy efficiency class } \\ \text { LS } & \text { Lower-standard energy efficiency class } \\ \text { p } & \text { Null-hypothesis probability (similarity) } \\ \text { R } 2 & \text { Coefficient of determination } \\ \text { RET } & \text { Renewable Energy Target } \\ \text { S } & \text { Standard energy efficiency class } \\ \text { SCA } & \text { Solar collector area } \\ \text { SCDi } & \text { Solar collector direction } \\ \text { SCDu } & \text { Solar collector dust accumulation } \\ \text { SCE } & \text { Solar collector efficiency } \\ \text { SCS } & \text { Solar collector shadowing } \\ \text { SCT } & \text { Solar collector tilt angle } \\ \text { SWH } & \text { Solar Water Heater } \\ \text { TKI } & \text { Tank heat loss } \\ \text { TKV } & \text { Tank volume } \\ \text { TLR6 } & \text { Toll-like receptor 6 } \\ \text { Upper-extreme energy efficiency class } \\ \text { Uper-standard energy efficiency class }\end{array}$

\section{Introduction}

Australia has one of the largest greenhouse gas (GHG) footprints among developed countries in the world due to its considerable reliance on fossil fuels for energy supply [1-3]. However, the country receives one of the greatest

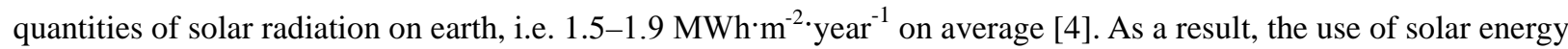
technologies has increased considerably (e.g. 7.2\% per annum from 2000 to 2008) in Australia in the last two decades in connection with the sanction of national policies on GHG emissions mitigation since 1997 [5]. These measures were usually implemented as part of government renewable energy rebate policies, e.g. Small-Scale Renewable Energy Scheme [3]. To date, the aim of such policies is to achieve at least $20 \%$ of electricity supply from renewable energy sources by 2020 as part of the Renewable Energy Target (RET) set by the Australian Federal Government, i.e. 33 TWh per year by 2020 [6,7]. 
Solar water heater (SWH) technologies were among the main strategies endorsed by renewable energy rebate schemes funded by the Australian government in the past decades. Since the 1950s, this technology has been researched and developed by Australian research and commercial institutions [8]. Notwithstanding developments in SWH technologies in the country, the proportion of the residential building stock with SWHs is approximately 5\% in Australia [3,4]. Traditionally, the most popular system is flat plate thermosiphon SWHs [4], but there has been an increase in the use of split SWHs.

In spite of general benefits associated with the use of SWHs in residences in Australia, the performance and electricity savings promoted by these systems is considerably influenced by the characteristics of their components, site-specific conditions and user behaviour. Therefore, SWHs may present inherent cost and efficiency limitations [2]. While cost limitations associated with SWHs for Australian households may be overcome with government renewable energy rebate schemes, efficiency limitations may persist if a careful selection of SWHs is not undertaken. As discussed by Mills [9], the performance of SWHs must be determined by using detailed models, rather than general approaches as the one defined by the Australian Standard AS 4234 - Heated water systems, calculation of energy consumption [10].

The assessment of SWHs must encompass a holistic approach based on multi-parametric analyses and associations between energy performance and service level indicators. Seldom are SWHs assessed considering a large range of variables and indicators under a single assessment framework. Nonetheless, recent studies on SWHs have addressed important aspects related to assessment frameworks, technological advancements, site-specific conditions, and user behaviour patterns. For instance, Allouhi et al. [11] indicated the importance of more efficient systems to reduce the reliance on energy from the electricity grid; Duomarco [12] pointed out the benefits of systems with high solar fractions; Halaway [13] alerted to the necessity of reviewing periodically the input assumption used to estimate the performance of SWHs, e.g. the standard AS/ANZ 4234:2008 on energy consumption modelling; Li et al. [14] showed that the application of vertical solar collectors are equally influence by site specific conditions, e.g. water consumption pattern and seasonal solar radiation; Yan et al. [15] demonstrated that considerable amounts of energy are missed due to a mismatch of solar collector energy supply and heating demand loads; Saravanan et al. [16] described the connection between technological advancements and performance improvements in the design of solar collectors; Tanha et al. [17] found that performance of systems is usually lower than the one described by manufacturers; Xue [18] studied the performance of new phase change materials for SWHs, which were significantly influenced by site conditions, e.g. cold water temperature and solar radiation variability; Lamnatou et al. [19] identified a lack of studies on the configuration of solar systems for building integrated design; Li et al. [20] investigated the impact of urban forms on the performance of solar systems, revealing a lower influence of site conditions (e.g. site coverage, building azimuths, etc.) on the performance of systems installed in low- and medium-density areas as opposed to high-density areas; Edwards et al. [21] suggested the use of variable water consumption loads based on empirical data, rather than static daily water consumption profiles, for performance assessment models of SWHs; Gill et al. [22] details in a qualitative analysis the interdependence among households, technologies and policies on SWHs, e.g. failure to achieve policy goals (household carbon emission reduction) and benefits for households (energy savings) due to a lack of quality professional advice and installation of systems; Lin et al. [23] assessed the financial feasibility of SWHs, which was dependent upon the daily water consumption pattern; and so on.

Notwithstanding the vast number of studies on SWHs, there is a dearth of studies that comprehensively integrates various technical specification parameters, site-specific conditions, and user behaviour aspects in order to holistically evaluate the performance of SWHs across multiple key performance indicators. The objective of the herein study is to assess the performance of SWHs for the city of Brisbane in Australia using a multi-parametric sensitivity analysis for technical specifications, site-specific conditions and user behaviour variables taking into account energy performance and service level indicators. The analysis was carried out considering the variability of 19 parameters and six performance indicators. 


\section{Research method}

This section provides an overview of the research method undertaken in this study. The study encompasses an analysis of SWHs focused on energy performance indicators (i.e. annual energy consumption, energy intensity, solar fraction and peak energy consumption) and level of service indicators (i.e. compliance with required hot water temperatures for Legionella spp. control and comfort levels). In order to accomplish the aim of the study, SWHs were assessed for the climate of the city of Brisbane considering 19 parameters combined into 192 scenarios emulated using calibrated computational models. Models were undertaken using the software EnergyPlus 8.6. The result of models were employed to analyse the influence of different technical specifications, site-specific conditions and user behaviour parameters on the six performance indicators of SWHs. Fig. 1 summarises the study method.

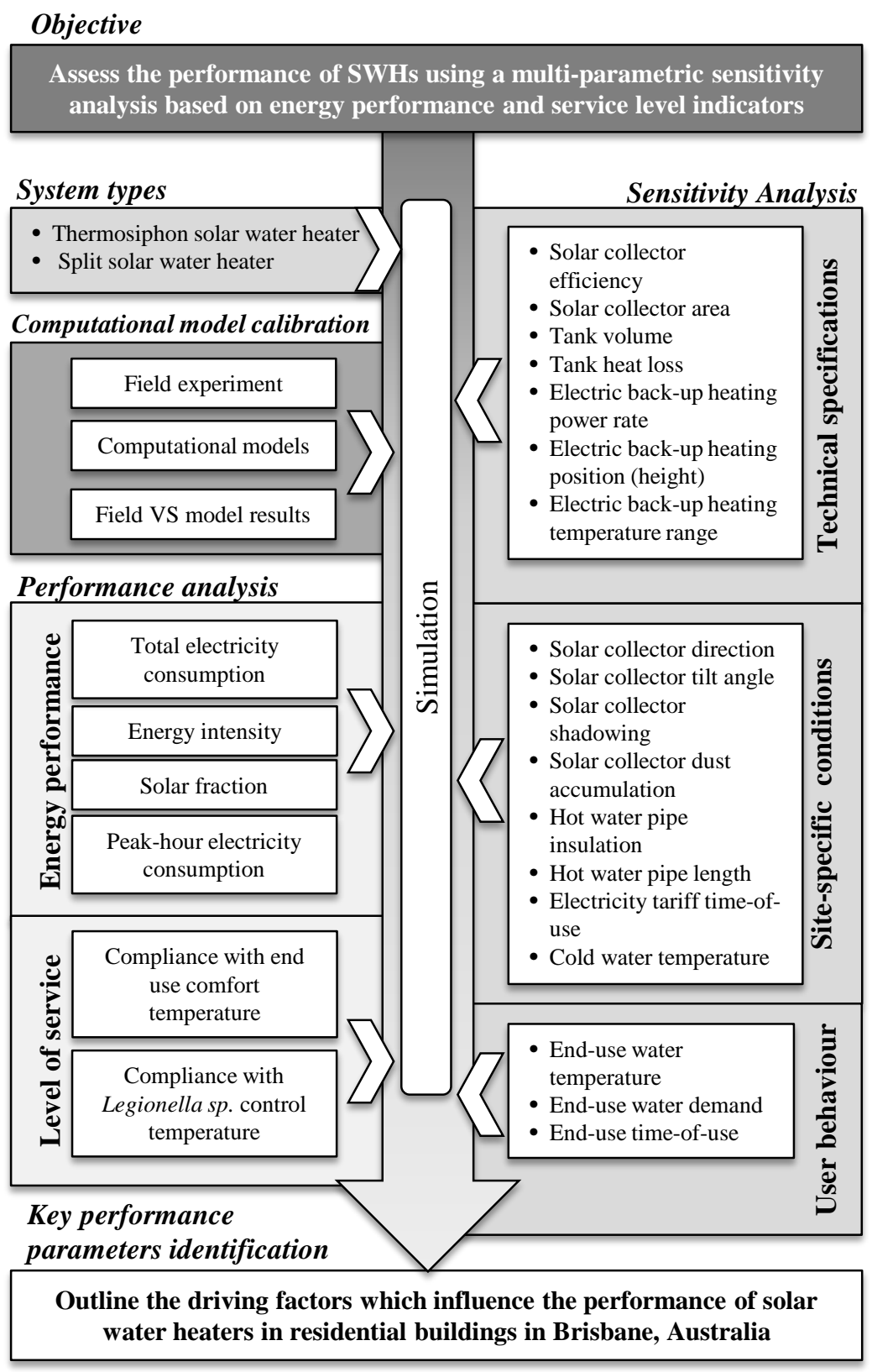

Fig. 1. Flow chart of the study method. 
In the supplementary material, a detailed explanation on supporting analyses is provided, including: (i) computational model calibration using a field experiment conducted in Brisbane, Australia (S-A); and (ii) review of input parameters for the sensitivity analysis (S-B).

\subsection{Sensitivity analysis inputs}

A sensitivity analysis was undertaken to evaluate the performance of split SWHs with vertical stratified tanks and thermosiphon SWHs with horizontal mixed tanks. Electricity was required during auxiliary heating events in split and thermosiphon systems alike, and for operating the pump of the circulation system between solar collectors and the tank in split systems only. In the analysis, three input categories were taken into account, namely: (i) technical specifications; (ii) site-specific conditions; and (iii) user behaviour.

The value range for most of the input parameters was based on five energy efficiency classes, i.e. lower-extreme (LE), lower-standard (LS), standard (S), upper-standard (US) and upper-extreme (UE). The variability of such values was statistically determined for stochastic parameters, whereas prior studies and expert appraisal were adopted for non-stochastic parameters range estimation (refer to Supplementary material S-B for more details). The performance analysis was undertaken considering the variation of values for single parameters at a time, while the other parameters were kept constant at the reference category. The reference category was fixed at the S class, except for the following parameters in accordance with the local solar market practices: solar collector efficiency at LS, solar collector direction at UE, solar collector tilt angle at UE, solar collector shadowing direction at UE, and tariff power interruption at LE. Therefore, the 19 parameters were combined into 192 scenarios by emulating eight directions for two parameters (solar collector orientation and shadowing, $n=16)$ and five values for other 16 parameters $(n=80)$, with a total of 96 scenarios for two system types (split and thermosiphon systems, $n=192$ ).

\subsection{Key performance indicators}

Each studied parameter was analysed considering performance indicators related to energy efficiency and level of service under a single framework. This approach provides a holistic analysis of SWHs, as conservative service levels may lead to excessive electricity consumption, whereas low energy consumption may be associated with reduced service levels.

Energy efficiency indicators encompassed the electricity consumption of auxiliary heating elements of SWHs, including the total annual electricity consumption as well as the peak-hour electricity consumption for the energy grid (i.e. from 4 $\mathrm{pm}$ to $8 \mathrm{pm}$ ). The total electricity consumption of SWHs was calculated by totalising the electric energy consumption of simulation scenarios for one year. The peak-hour electricity consumption was calculated in an analogous way to the total electricity consumption, but considering peak-hours only.

The solar fraction of SWHs was also used as an indicator. It was calculated as the quotient of the amount of solar energy to the total energy consumption of SWHs (i.e. solar energy and electricity). The consumed solar energy was calculated as a function of the subtraction between the total energy consumption and the electricity requirements of SWHs. Moreover, energy efficiency indicators also included electric energy intensity (unit of energy per unit of water consumption).

Service indicators were related to compliance levels with temperature thresholds for comfort level of users at end-use points and protection against Legionella spp. growth in hot water tanks. The compliance level with the end-use point temperatures was analysed by comparing the supplied water temperature with their set-point temperature. The required 
criteria for Legionella spp. control was adopted based on a continuous supply temperature at the storage tank outlet (top hot water layer) of $60{ }^{\circ} \mathrm{C}$ as suggested by Bédard et al. [24] and Boppe et al. [25] among other current studies published by government agencies (e.g. UK [26]). Notwithstanding the general inhibition of Legionella spp. for temperature equal to $60{ }^{\circ} \mathrm{C}$, such bacteria group may persist under growth-restrictive temperatures $\left(<20\right.$ and $\left.>55^{\circ} \mathrm{C}\right)$ and heat shocks $\left(70^{\circ} \mathrm{C}\right.$ for 30 minutes) [27].

The herein assessment of Legionella spp. control temperature only provides an indication of the susceptibility of hot water supply systems to Legionella spp. growth as described in Table 1. Mercante and Winchell [27] pointed out that the proliferation of Legionella spp. in hot water supply systems is not directly associated with clinical cases of legionellosis, as this disease tend to occur when other underlying factors in addition to exposure to aerosols with Legionella spp. are present, such as: over 50 years-old man with lung disease, immunosuppression, smoking, recent travel, diabetes, cancer, acquired immune deficiency syndrome, end-stage renal disease, and human cellular Toll-like receptor 6 (TLR6) mutations. The authors also reported that the number of legionellosis has increased in the past decades, which may be attributed to a rise in diagnostic testing techniques, improvements in reporting, and growth of vulnerable elderly population.

Table 1. Legionella spp. proliferation risk in hot water supply systems.

\begin{tabular}{|l|l|l|}
\hline Water heater outlet temperature $\left({ }^{\circ} \mathrm{C}\right)$ & Time frequency (\%) & Risk \\
\hline$\geq 60$ & 100 & Very low \\
\hline$\geq 60$ & $90-99$ & Low \\
\hline$\geq 60$ & $51-89$ & Intermediate \\
\hline$\geq 60$ & $0-50$ & High \\
\hline
\end{tabular}

Note: Adapted from Bédard et al. [24].

\section{Performance analysis}

Among the 19 studied parameters, the sensitivity analysis revealed a diversified interaction among the performance of SWHs, both in terms of energy efficiency and level of service, and the variability of technical specifications of SWHs, site specific conditions and user behaviour. As shown in Table 2, the electricity consumption of SWHs underwent a significant variation under different energy efficiency classes for most of the parameters.

Table 2. Absolute and relative change in electricity consumption.

\begin{tabular}{|c|c|c|c|c|c|c|c|c|c|c|c|}
\hline \multirow{2}{*}{\multicolumn{2}{|c|}{$\begin{array}{c}\text { System type } \\
\text { Energy efficiency class }\end{array}$}} & \multicolumn{5}{|c|}{ Split with stratified vertical tank } & \multicolumn{5}{|c|}{ Thermosiphon with mixed horizontal tank } \\
\hline & & $\mathbf{L E}$ & LS & $\mathbf{S}$ & US & UE & $\mathbf{L E}$ & LS & $\mathbf{S}$ & US & UE \\
\hline \multirow{18}{*}{ 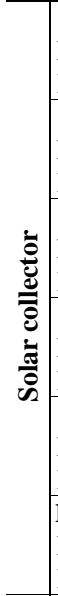 } & Efficiency (\%) & 39 & 43 & 56 & 64 & 67 & 39 & 43 & 56 & 64 & 67 \\
\hline & Electricity use (kWh/year) & 416 & 340 & 118 & 107 & 100 & 450 & 365 & 61 & 42 & 32 \\
\hline & Electricity use change (\%) & 22 & 0 & -65 & -68 & -71 & 23 & 0 & -83 & -88 & -91 \\
\hline & Area $\left(\mathrm{m}^{2}\right)$ & 1.8 & 2.8 & 5.6 & 9.0 & 10.4 & 1.8 & 2.8 & 5.6 & 9.0 & 10.4 \\
\hline & Electricity use (kWh/year) & 1072 & 749 & 340 & 201 & 176 & 875 & 643 & 365 & 236 & 219 \\
\hline & Electricity use change (\%) & 215 & 120 & 0 & -41 & -48 & 140 & 76 & 0 & -36 & -40 \\
\hline & Direction/orientation* & $\mathrm{S}$ & SE/SW & $\mathrm{E} / \mathrm{W}$ & NE/NW & $\mathrm{N}$ & $\mathrm{S}$ & SE/SW & $\mathrm{E} / \mathrm{W}$ & $\mathrm{NE} / \mathrm{NW}$ & $\mathrm{N}$ \\
\hline & Electricity use (kWh/year) & 1099 & 940 & 614 & 397 & 340 & 1023 & 893 & 662 & 438 & 365 \\
\hline & Electricity use change (\%) & 223 & 177 & 80 & 17 & 0 & 180 & 144 & 81 & 20 & 0 \\
\hline & Tilt angle & $90^{\circ}$ & $0^{\circ}$ & $13.7^{\circ}$ & $47.4^{\circ}$ & $27.4^{\circ}$ & $90^{\circ}$ & $0^{\circ}$ & $13.7^{\circ}$ & $47.4^{\circ}$ & $27.4^{\circ}$ \\
\hline & Electricity use (kWh/year) & 1314 & 609 & 437 & 381 & 340 & 1270 & - & 489 & 401 & 365 \\
\hline & Electricity use change (\%) & 286 & 79 & 29 & 12 & 0 & 248 & - & 34 & 10 & 0 \\
\hline & Shadowing direction* & $\mathrm{N}$ & $\mathrm{NE} / \mathrm{NW}$ & E/W & $\mathrm{S} / \mathrm{SE} / \mathrm{SW}$ & None & $\mathrm{N}$ & NE/NW & $\mathrm{E} / \mathrm{W}$ & S/SE/SW & None \\
\hline & Electricity use (kWh/year) & 586 & 447 & 354 & 347 & 340 & 642 & 509 & 394 & 369 & 365 \\
\hline & Electricity use change (\%) & 72 & 31 & 4 & 2 & 0 & 76 & 39 & 8 & 1 & 0 \\
\hline & Dust radiation decline (\%) & 64.0 & 47.0 & 5.0 & 2.7 & 0.0 & 64.0 & 47.0 & 5.0 & 2.7 & 0.0 \\
\hline & Electricity use (kWh/year) & 1584 & 1234 & 340 & 312 & 283 & 1449 & 1235 & 365 & 332 & 294 \\
\hline & Electricity use change (\%) & 366 & 263 & 0 & -8 & -17 & 297 & 238 & 0 & -9 & -19 \\
\hline \multirow{4}{*}{ 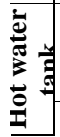 } & Volume (litres) & 90 & 250 & 315 & 450 & 630 & 90 & 250 & 315 & 450 & 630 \\
\hline & Electricity use (kWh/year) & 549 & 372 & 340 & 354 & 385 & 774 & 434 & 365 & 291 & 240 \\
\hline & Electricity use change (\%) & 62 & 10 & 0 & 4 & 13 & 112 & 19 & 0 & -20 & -34 \\
\hline & \begin{tabular}{|l|} 
Heat loss (W/K) \\
\end{tabular} & 3.1 & 1.3 & 0.8 & 0.5 & 0.2 & 3.1 & 1.3 & 0.8 & 0.5 & 0.2 \\
\hline
\end{tabular}




\begin{tabular}{|c|c|c|c|c|c|c|c|c|c|c|c|}
\hline & Electricity use (kWh/year) & 1569 & 605 & 340 & 241 & 165 & 963 & 507 & 365 & 303 & 246 \\
\hline & Electricity use change (\%) & 361 & 78 & 0 & -29 & -51 & 163 & 39 & 0 & -17 & -33 \\
\hline & Cold water connection $\left({ }^{\circ} \mathrm{C}\right)$ & $14-21$ & $16-23$ & $20-27$ & $24-31$ & $26-33$ & $14-21$ & $16-23$ & $20-27$ & $24-31$ & $26-33$ \\
\hline & Electricity use (kWh/year) & 517 & 452 & 340 & 248 & 212 & 636 & 544 & 365 & 213 & 160 \\
\hline & Electricity use change (\%) & 52 & 33 & 0 & -27 & -38 & 74 & 49 & 0 & -42 & -56 \\
\hline g & Insulation (R-value) & 0.0 & 0.2 & 0.3 & 1.0 & $\mathrm{AD}^{* *}$ & 0.0 & 0.2 & 0.3 & 1.0 & $\mathrm{AD}^{* *}$ \\
\hline 茨 & Electricity use (kWh/year) & 433 & 343 & 340 & 339 & 297 & 406 & 368 & 365 & 362 & 270 \\
\hline 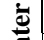 & Electricity use change (\%) & 27 & 1 & 0 & 0 & -13 & 11 & 1 & 0 & -1 & -26 \\
\hline$\frac{\pi}{3}$ & Length (metres) & 18 & 15 & 9 & 5 & 0 & 18 & 15 & 9 & 5 & 0 \\
\hline 5 & Electricity use (kWh/year) & 364 & 356 & 340 & 331 & 298 & 411 & 402 & 365 & 326 & 270 \\
\hline $\bar{\Psi}$ & Electricity use change (\%) & 7 & 5 & 0 & -3 & -12 & 13 & 10 & 0 & -11 & -26 \\
\hline & Element power (kW) & 5.5 & 4.8 & 3.0 & 1.5 & 0.9 & 5.5 & 4.8 & 3.0 & 1.5 & 0.9 \\
\hline & Electricity use (kWh/year) & 325 & 325 & 340 & 348 & 328 & 367 & 367 & 365 & 352 & 334 \\
\hline & Electricity use change (\%) & -4 & -4 & 0 & 2 & -3 & 1 & 0 & 0 & -4 & -9 \\
\hline. & Element tank height (\%) & 0 & 25 & 50 & 75 & 100 & - & - & - & - & - \\
\hline 氙 & Electricity use (kWh/year) & 1029 & 460 & 340 & 280 & 245 & - & - & - & - & - \\
\hline$\frac{\pi}{5}$ & Electricity use change (\%) & 203 & 35 & 0 & -18 & -28 & - & - & - & - & - \\
\hline . & Set-point temperature $\left({ }^{\circ} \mathrm{C}\right)$ & 80 & 74 & 60 & 53 & 50 & 80 & 74 & 60 & 53 & 50 \\
\hline ]్ర & Electricity use (kWh/year) & 1334 & 965 & 340 & 182 & 143 & 1195 & 918 & 365 & 173 & 137 \\
\hline 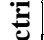 & Electricity use change (\%) & 292 & 184 & 0 & -47 & -58 & 227 & 151 & 0 & -53 & -62 \\
\hline$\frac{\mathscr{Q}}{\sigma T}$ & Tariff power interruption (h) & 0 & 2 & 3 & 6 & 16 & 0 & 2 & 3 & 6 & 16 \\
\hline & Electricity use (kWh/year) & 340 & 337 & 337 & 320 & 175 & 365 & 365 & 365 & 321 & 236 \\
\hline & Electricity use change (\%) & 0 & -1 & -1 & -6 & -49 & 0 & 0 & 0 & -12 & -35 \\
\hline & End-use temperature $\left({ }^{\circ} \mathrm{C}\right)$ & 50 & 47 & 40 & 35 & 33 & 50 & 47 & 40 & 35 & 33 \\
\hline & Electricity use (kWh/year) & 571 & 517 & 340 & 239 & 206 & 814 & 664 & 365 & 192 & 129 \\
\hline$\Xi$ & Electricity use change (\%) & 68 & 52 & 0 & -30 & -39 & 123 & 82 & 0 & -48 & -65 \\
\hline. & Water demand (litres/day) & 233 & 210 & 155 & 100 & 77 & 233 & 210 & 155 & 100 & 77 \\
\hline E & Electricity use (kWh/year) & 612 & 521 & 340 & 208 & 162 & 815 & 664 & 365 & 138 & 88 \\
\hline 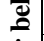 & Electricity use change (\%) & 80 & 53 & 0 & -39 & -52 & 123 & 82 & 0 & -62 & -76 \\
\hline 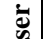 & Time of peak use hour (24h) & $6: 50$ & $7 \& 17$ & $7: 30 \& 18$ & $9 \& 20$ & 9:40 & $6: 50$ & $7 \& 17$ & $7: 30 \& 18$ & $9 \& 20$ & $9: 40$ \\
\hline $\mathscr{n}$ & Electricity use (kWh/year) & 350 & 342 & 340 & 291 & 248 & 349 & 364 & 365 & 334 & 300 \\
\hline & Electricity use change (\%) & 3 & 0 & 0 & -14 & -27 & -5 & 0 & 0 & -8 & -18 \\
\hline
\end{tabular}

Note: * North (N), Northeast (NE), Northwest (NW), East (E), West (W), Southeast (SE), Southwest (SW), South (S); ** Adiabatic $(A D)$

Under the reference energy efficiency class for each parameter (base scenario), split and thermosiphon SWHs had an electricity consumption of 340 and $365 \mathrm{kWh} \cdot y e a r^{-1}$ per household, respectively, with an equivalent energy intensity of approximately $6 \mathrm{kWh} \cdot \mathrm{m}^{-3}$. The use other energy efficiency classes showed a significant effect on the electricity savings of SWHs. For instance, the replacement of lower-standard collectors (most popular collector category in the local market) with standard performance collectors (median efficiency level among the 135 glazed solar collectors' sample) promoted a decrease in energy intensity of $65 \%\left(1 \mathrm{kWh} \cdot \mathrm{m}^{-3}\right)$ for split systems and $83 \%\left(2 \mathrm{kWh} \cdot \mathrm{m}^{-3}\right)$ for thermosiphon systems. Conversely, a 64\% transmittance reduction on solar collectors associated with excessive levels of dust accumulation, or other type of dirt (e.g. grime, bird drops, etc.), may almost eliminate the solar energy collection of SWHs. Under such conditions, there exists a tremendous increase in electricity consumption and associated energy intensity to ranges comparable to electric water heaters, i.e. 25 and $28 \mathrm{kWh} \cdot \mathrm{m}^{-3}$ for thermosiphon and split SWHs, respectively.

Reductions in the electricity consumption during peak-hours for the energy grid (i.e. from $4 \mathrm{pm}$ to $8 \mathrm{pm}$ ) were mainly related to two major SWHs groups, namely: (i) systems with high energy efficiency and low electricity requirements during peak-hours; and (ii) systems with low energy efficiency with a random displacement of electricity consumption from peak-hours. The peak electricity consumption analysis is summarised in Table 3.

Table 3. Absolute and relative change in peak electricity consumption.

\begin{tabular}{|c|c|c|c|c|c|c|c|c|c|c|c|}
\hline \multirow{2}{*}{\multicolumn{2}{|c|}{$\begin{array}{c}\text { System type } \\
\text { Energy efficiency class }\end{array}$}} & \multicolumn{5}{|c|}{ Split with stratified vertical tank } & \multicolumn{5}{|c|}{ Thermosiphon with mixed horizontal tank } \\
\hline & & $\mathbf{L E}$ & LS & $\mathbf{S}$ & US & UE & $\mathbf{L E}$ & LS & $\mathbf{S}$ & US & UE \\
\hline \multirow{10}{*}{ 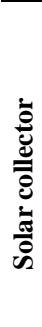 } & Efficiency (\%) & 39 & 43 & 56 & 64 & 67 & 39 & 43 & 56 & 64 & 67 \\
\hline & Peak use (kWh/year) & 12 & 17 & 6 & 3 & 0 & 72 & 91 & 15 & 8 & 8 \\
\hline & Peak use change (\%) & -27 & 0 & -65 & -81 & -100 & -21 & 0 & -83 & -92 & -92 \\
\hline & Area $\left(\mathrm{m}^{2}\right)$ & 1.8 & 2.8 & 5.6 & 9 & 10.4 & 1.8 & 2.8 & 5.6 & 9 & 10.4 \\
\hline & Peak use (kWh/year) & 32 & 22 & 17 & 10 & 7 & 114 & 109 & 91 & 80 & 79 \\
\hline & Peak use change (\%) & 89 & 32 & 0 & -41 & -59 & 25 & 20 & 0 & -12 & -14 \\
\hline & Direction/orientation* & $S$ & SE/SW & $\mathrm{E} / \mathrm{W}$ & $\mathrm{NE} / \mathrm{NW}$ & $\mathrm{N}$ & $S$ & SE/SW & $\mathrm{E} / \mathrm{W}$ & NE/NW & $\mathrm{N}$ \\
\hline & Peak use (kWh/year) & 207 & 155 & 25 & 16 & 18 & 49 & 387 & 67 & 58 & 91 \\
\hline & Peak use change (\%) & 1023 & 738 & 36 & -15 & 0 & -46 & 323 & -27 & -36 & 0 \\
\hline & Tilt angle & $90^{\circ}$ & $0^{\circ}$ & $13.7^{\circ}$ & $47.4^{\circ}$ & $27.4^{\circ}$ & $90^{\circ}$ & $0^{\circ}$ & $13.7^{\circ}$ & $47.4^{\circ}$ & $27.4^{\circ}$ \\
\hline
\end{tabular}




\begin{tabular}{|c|c|c|c|c|c|c|c|c|c|c|c|}
\hline & $\begin{array}{l}\text { Peak use (kWh/year) } \\
\text { Peak use change }(\%)\end{array}$ & $\begin{array}{l}162 \\
869\end{array}$ & $\begin{array}{c}17 \\
0\end{array}$ & $\begin{array}{l}13 \\
-21\end{array}$ & $\begin{array}{l}15 \\
-12\end{array}$ & $\begin{array}{c}17 \\
0\end{array}$ & $\begin{array}{l}318 \\
247\end{array}$ & $\begin{array}{l}- \\
-\end{array}$ & $\begin{array}{l}31 \\
-66\end{array}$ & $\begin{array}{c}128 \\
40\end{array}$ & $\begin{array}{c}91 \\
0\end{array}$ \\
\hline & Shadowing direction* & $\mathrm{N}$ & $\mathrm{NE} / \mathrm{NW}$ & $\mathrm{E} / \mathrm{W}$ & S/SE/SW & None & $\mathrm{N}$ & $\mathrm{NE} / \mathrm{NW}$ & $\mathrm{E} / \mathrm{W}$ & $\mathrm{S} / \mathrm{SE} / \mathrm{SW}$ & None \\
\hline & Peak use (kWh/year) & 32 & 25 & 19 & 18 & 17 & 48 & 93 & 91 & 91 & 91 \\
\hline & Peak use change (\%) & 89 & 48 & 13 & 3 & 0 & -48 & 1 & -1 & 0 & 0 \\
\hline & Dust radiation decline (\%) & 64 & 47 & 5 & 2.7 & 0 & 64 & 47 & 5 & 2.7 & 0 \\
\hline & Peak use (kWh/year) & 172 & 59 & 18 & 15 & 16 & 6 & 80 & 91 & 82 & 79 \\
\hline & Peak use change (\%) & 833 & 219 & 0 & -16 & -13 & -93 & -13 & 0 & -11 & -14 \\
\hline \multirow{9}{*}{ 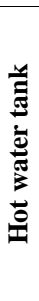 } & Volume (litres) & 90 & 250 & 315 & 450 & 630 & 90 & 250 & 315 & 450 & 630 \\
\hline & Peak use (kWh/year) & 43 & 28 & 18 & 21 & 41 & 172 & 71 & 91 & 94 & 67 \\
\hline & Peak use change (\%) & 134 & 54 & 0 & 12 & 122 & 88 & -23 & 0 & 2 & -27 \\
\hline & Heat loss (W/K) & 3.08 & 1.32 & 0.75 & 0.47 & 0.18 & 3.08 & 1.32 & 0.75 & 0.47 & 0.18 \\
\hline & Peak use (kWh/year) & 125 & 36 & 17 & 10 & 5 & 164 & 61 & 91 & 88 & 96 \\
\hline & Peak use change (\%) & 638 & 113 & 0 & -43 & -71 & 79 & -33 & 0 & -4 & 5 \\
\hline & Cold water connection $\left({ }^{\circ} \mathrm{C}\right)$ & $14-21$ & $16-23$ & $20-27$ & $24-31$ & $26-33$ & $14-21$ & $16-23$ & $20-27$ & $24-31$ & $26-33$ \\
\hline & Peak use (kWh/year) & 12 & 15 & 17 & 9 & 7 & 118 & 78 & 91 & 63 & 52 \\
\hline & Peak use change (\%) & -27 & -11 & 0 & -44 & -58 & 29 & -15 & 0 & -32 & -43 \\
\hline \multirow{6}{*}{ 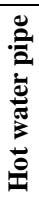 } & Insulation (R-value) & 0 & 0.2 & 0.3 & 1 & $\mathrm{AD}^{* *}$ & 0 & 0.2 & 0.3 & 1 & $\mathrm{AD}^{* *}$ \\
\hline & Peak use (kWh/year) & 29 & 19 & 18 & 18 & 12 & 85 & 92 & 91 & 88 & 90 \\
\hline & Peak use change $(\%)$ & 56 & 3 & 0 & -4 & -33 & -7 & 0 & 0 & -4 & -2 \\
\hline & Length (metres) & 18 & 15 & 9 & 5 & 0 & 18 & 15 & 9 & 5 & 0 \\
\hline & Peak use (kWh/year) & 15 & 18 & 17 & 17 & 12 & 86 & 68 & 91 & 78 & 89 \\
\hline & Peak use change (\%) & -14 & 5 & 0 & -3 & -30 & -5 & -25 & 0 & -14 & -2 \\
\hline \multirow{12}{*}{ 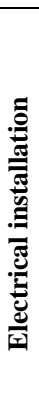 } & Element power (kW) & 5.5 & 4.8 & 3 & 1.5 & 0.9 & 5.5 & 4.8 & 3 & 1.5 & 0.9 \\
\hline & Peak use (kWh/year) & 26 & 27 & 18 & 18 & 19 & 91 & 91 & 91 & 91 & 32 \\
\hline & Peak use change (\%) & 42 & 44 & 0 & -1 & 2 & 0 & 0 & 0 & -1 & -65 \\
\hline & Element tank height (\%) & 0 & 25 & 50 & 75 & 100 & - & - & - & - & - \\
\hline & Peak use (kWh/year) & 381 & 83 & 17 & 6 & 10 & - & - & - & - & - \\
\hline & Peak use change (\%) & 2140 & 387 & 0 & -67 & -42 & - & - & - & - & - \\
\hline & Set-point temperature $\left({ }^{\circ} \mathrm{C}\right)$ & 80 & 74 & 60 & 53 & 50 & 80 & 74 & 60 & 53 & 50 \\
\hline & Peak use (kWh/year) & 67 & 48 & 17 & 11 & 4 & 323 & 248 & 91 & 45 & 25 \\
\hline & Peak use change (\%) & 292 & 184 & 0 & -36 & -75 & 253 & 171 & 0 & -51 & -73 \\
\hline & Tariff power interruption (h) & 0 & 2 & 3 & 6 & 16 & 0 & 2 & 3 & 6 & 16 \\
\hline & Peak use (kWh/year) & 17 & 3 & 0 & 0 & 0 & 91 & 1 & 0 & 0 & 0 \\
\hline & Peak use change (\%) & 0 & -84 & -100 & -100 & -100 & 0 & -98 & -100 & -100 & -100 \\
\hline \multirow{9}{*}{ 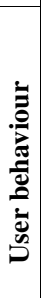 } & End-use temperature $\left({ }^{\circ} \mathrm{C}\right)$ & 50 & 47 & 40 & 35 & 33 & 50 & 47 & 40 & 35 & 33 \\
\hline & Peak use (kWh/year) & 33 & 21 & 17 & 8 & 3 & 147 & 129 & 91 & 38 & 48 \\
\hline & Peak use change (\%) & 97 & 28 & 0 & -53 & -80 & 61 & 42 & 0 & -59 & -47 \\
\hline & Water demand (litres/day) & 233 & 210 & 155 & 100 & 77 & 233 & 210 & 155 & 100 & 77 \\
\hline & Peak use (kWh/year) & 33 & 22 & 17 & 5 & 6 & 116 & 109 & 91 & 49 & 28 \\
\hline & Peak use change (\%) & 95 & 31 & 0 & -71 & -66 & 27 & 19 & 0 & -46 & -69 \\
\hline & Time of peak use hour (24h) & $6: 50$ & $7 \& 17$ & $7: 30 \& 18$ & $9 \& 20$ & $9: 40$ & $6: 50$ & $7 \& 17$ & $7: 30 \& 18$ & $9 \& 20$ & $9: 40$ \\
\hline & Peak use (kWh/year) & 5 & 17 & 17 & 4 & 3 & 0 & 98 & 91 & 3 & 0 \\
\hline & Peak use change (\%) & -71 & 1 & 0 & -74 & -83 & -100 & 7 & 0 & -97 & -100 \\
\hline
\end{tabular}

Note: * North (N), Northeast (NE), Northwest (NW), East (E), West (W), Southeast (SE), Southwest (SW), South (S); ** Adiabatic $(A D)$

The use of high energy performance systems was associated with a significant decrease in peak-hour electricity requirements, e.g. total displacement of electricity from peak-hours by adopting split SWHs with upper-extreme solar collector efficiency. This outcome has a direct benefit for the energy sector, as a reduction in peak-hour electricity consumption usually contributes to a deferral of capital expenditures for upgrades of energy grids. Peak-hour electricity reductions were also achieved by systems with low performance (e.g. thermosiphon SWHs with North shadowing or 64\% transmittance reduction due to dust accumulation in collectors). Notwithstanding this apparent benefit, such systems have a limited electricity saving potential and may lead to a considerable increase in the peak-hour energy consumption depending on user behaviour pattern. The use of split SWHs with electric heating element placed at the bottom of hot water tanks showed the largest increase in peak-hour electricity consumption (21-fold) in relation to the base scenario. This system configuration (i.e. heating element and thermostat positioned at the same level as the cold water inlet) operates constantly heating cold water, and hence provides a larger hot water supply capacity by jeopardising the potential of split SWHs to divert electricity consumption from peak-hours.

The solar energy use proportion in relation to the total energy requirement of SWHs (solar fraction) was mainly influenced by factors associated with the characteristics of solar collectors (Table 4); however, other factors related to user behaviour and back-up electricity heating set-point temperature also played an important role in the solar fraction of SWHs. 
Table 4. Absolute and relative change in solar energy fraction.

\begin{tabular}{|c|c|c|c|c|c|c|c|c|c|c|c|}
\hline \multirow{2}{*}{\multicolumn{2}{|c|}{$\begin{array}{c}\text { System type } \\
\text { Energy efficiency class }\end{array}$}} & \multicolumn{5}{|c|}{ Split with stratified vertical tank } & \multicolumn{5}{|c|}{ Thermosiphon with mixed horizontal tank } \\
\hline & & $\mathbf{L E}$ & LS & $\mathbf{S}$ & US & UE & $\mathbf{L E}$ & LS & $\mathbf{S}$ & US & UE \\
\hline \multirow{18}{*}{ 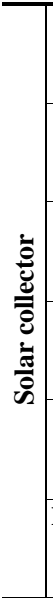 } & Efficiency (\%) & 39 & 43 & 56 & 64 & 67 & 39 & 43 & 56 & 64 & 67 \\
\hline & Solar fraction (\%) & 82 & 86 & 97 & 98 & 98 & 71 & 76 & 97 & 98 & 98 \\
\hline & Solar fraction change (\%) & -5 & 0 & 13 & 14 & 14 & -7 & 0 & 28 & 29 & 29 \\
\hline & Area $\left(\mathrm{m}^{2}\right)$ & 1.8 & 2.8 & 5.6 & 9 & 10.4 & 1.8 & 2.8 & 5.6 & 9 & 10.4 \\
\hline & Solar fraction (\%) & 45 & 64 & 86 & 93 & 94 & 41 & 57 & 76 & 85 & 86 \\
\hline & Solar fraction change (\%) & -48 & -26 & 0 & 8 & 9 & -46 & -25 & 0 & 12 & 13 \\
\hline & Direction/orientation* & $\mathrm{S}$ & SE/SW & $\mathrm{E} / \mathrm{W}$ & $\mathrm{NE} / \mathrm{NW}$ & $\mathrm{N}$ & $\mathrm{S}$ & SE/SW & E/W & $\mathrm{NE} / \mathrm{NW}$ & $\mathrm{N}$ \\
\hline & Solar fraction (\%) & 42 & 52 & 71 & 83 & 86 & 31 & 40 & 57 & 72 & 76 \\
\hline & Solar fraction change (\%) & -51 & -39 & -17 & -4 & 0 & -59 & -47 & -26 & -6 & 0 \\
\hline & Tilt angle & $90^{\circ}$ & $0^{\circ}$ & $13.7^{\circ}$ & $47.4^{\circ}$ & $27.4^{\circ}$ & $90^{\circ}$ & $0^{\circ}$ & $13.7^{\circ}$ & $47.4^{\circ}$ & $27.4^{\circ}$ \\
\hline & Solar fraction (\%) & 29 & 72 & 81 & 83 & 86 & 14 & - & 68 & 74 & 76 \\
\hline & Solar fraction change (\%) & -67 & -17 & -6 & -3 & 0 & -82 & - & -10 & -4 & 0 \\
\hline & Shadowing direction* & $\mathrm{N}$ & $\mathrm{NE} / \mathrm{NW}$ & E/W & S/SE/SW & None & $\mathrm{N}$ & NE/NW & E/W & S/SE/SW & None \\
\hline & Solar fraction (\%) & 73 & 79 & 85 & 85 & 86 & 58 & 67 & 74 & 76 & 76 \\
\hline & Solar fraction change (\%) & -16 & -7 & -1 & 0 & 0 & -24 & -13 & -3 & 0 & 0 \\
\hline & Dust radiation decline (\%) & 64 & 47 & 5 & 2.7 & 0 & 64 & 47 & 5 & 2.7 & 0 \\
\hline & Solar fraction (\%) & 13 & 35 & 86 & 87 & 89 & 1 & 16 & 76 & 79 & 81 \\
\hline & Solar fraction change (\%) & -85 & -59 & 0 & 2 & 3 & -99 & -79 & 0 & 3 & 6 \\
\hline \multirow{9}{*}{ 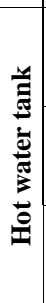 } & Volume (litres) & 90 & 250 & 315 & 450 & 630 & 90 & 250 & 315 & 450 & 630 \\
\hline & Solar fraction (\%) & 69 & 83 & 86 & 86 & 86 & 50 & 72 & 76 & 81 & 84 \\
\hline & Solar fraction change (\%) & -20 & -3 & 0 & 1 & 1 & -35 & -6 & 0 & 6 & 10 \\
\hline & Heat loss (W/K) & 3.08 & 1.32 & 0.75 & 0.47 & 0.18 & 3.08 & 1.32 & 0.75 & 0.47 & 0.18 \\
\hline & Solar fraction (\%) & 60 & 78 & 86 & 89 & 92 & 59 & 71 & 76 & 79 & 82 \\
\hline & Solar fraction change (\%) & -30 & -9 & 0 & 3 & 7 & -22 & -7 & 0 & 4 & 8 \\
\hline & Cold water connection $\left({ }^{\circ} \mathrm{C}\right)$ & $14-21$ & $16-23$ & $20-27$ & $24-31$ & $26-33$ & $14-21$ & $16-23$ & $20-27$ & $24-31$ & $26-33$ \\
\hline & Solar fraction (\%) & 81 & 82 & 86 & 89 & 90 & 67 & 70 & 76 & 84 & 87 \\
\hline & Solar fraction change (\%) & -6 & -4 & 0 & 4 & 5 & -12 & -9 & 0 & 9 & 13 \\
\hline \multirow{6}{*}{ 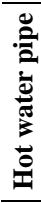 } & Insulation (R-value) & 0 & 0.2 & 0.3 & 1 & $\mathrm{AD}^{* *}$ & 0 & 0.2 & 0.3 & 1 & $\mathrm{AD}^{* *}$ \\
\hline & Solar fraction (\%) & 83 & 86 & 86 & 86 & 87 & 75 & 76 & 76 & 77 & 81 \\
\hline & Solar fraction change (\%) & -4 & 0 & 0 & 0 & 2 & -2 & 0 & 0 & 0 & 6 \\
\hline & Length (metres) & 18 & 15 & 9 & 5 & 0 & 18 & 15 & 9 & 5 & 0 \\
\hline & Solar fraction (\%) & 85 & 85 & 86 & 86 & 87 & 75 & 75 & 76 & 78 & 81 \\
\hline & Solar fraction change (\%) & -1 & -1 & 0 & 0 & 1 & -1 & -1 & 0 & 3 & 7 \\
\hline \multirow{12}{*}{ 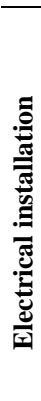 } & Element power (kW) & 5.5 & 4.8 & 3 & 1.5 & 0.9 & 5.5 & 4.8 & 3 & 1.5 & 0.9 \\
\hline & Solar fraction (\%) & 87 & 87 & 86 & 85 & 86 & 76 & 76 & 76 & 77 & 78 \\
\hline & Solar fraction change (\%) & 1 & 1 & 0 & 0 & 1 & 0 & 0 & 0 & 1 & 3 \\
\hline & Element tank height (\%) & 0 & 25 & 50 & 75 & 100 & - & - & - & - & - \\
\hline & Solar fraction (\%) & 54 & 80 & 86 & 89 & 90 & - & - & - & - & - \\
\hline & Solar fraction change (\%) & -37 & -7 & 0 & 3 & 5 & - & - & - & - & - \\
\hline & Set-point temperature $\left({ }^{\circ} \mathrm{C}\right)$ & 80 & 74 & 60 & 53 & 50 & 80 & 74 & 60 & 53 & 50 \\
\hline & Solar fraction (\%) & 42 & 58 & 86 & 94 & 96 & 29 & 44 & 76 & 89 & 91 \\
\hline & Solar fraction change (\%) & -51 & -33 & 0 & 9 & 12 & -62 & -42 & 0 & 17 & 20 \\
\hline & Tariff power interruption (h) & 0 & 2 & 3 & 6 & 16 & 0 & 2 & 3 & 6 & 16 \\
\hline & Solar fraction (\%) & 86 & 86 & 86 & 87 & 94 & 76 & 76 & 76 & 79 & 85 \\
\hline & Solar fraction change (\%) & 0 & 0 & 0 & 1 & 9 & 0 & 0 & 0 & 4 & 11 \\
\hline \multirow{9}{*}{ 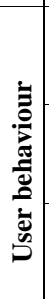 } & End-use temperature $\left({ }^{\circ} \mathrm{C}\right)$ & 50 & 47 & 40 & 35 & 33 & 50 & 47 & 40 & 35 & 33 \\
\hline & Solar fraction (\%) & 79 & 81 & 86 & 89 & 90 & 62 & 66 & 76 & 85 & 89 \\
\hline & Solar fraction change (\%) & -7 & -6 & 0 & 4 & 5 & -19 & -13 & 0 & 11 & 16 \\
\hline & Water demand (litres/day) & 233 & 210 & 155 & 100 & 77 & 233 & 210 & 155 & 100 & 77 \\
\hline & Solar fraction (\%) & 78 & 81 & 86 & 91 & 93 & 61 & 66 & 76 & 88 & 91 \\
\hline & Solar fraction change (\%) & -9 & -6 & 0 & 6 & 8 & -20 & -14 & 0 & 16 & 20 \\
\hline & Time of peak use hour (24h) & $6: 50$ & $7 \& 17$ & $7: 30 \& 18$ & $9 \& 20$ & $9: 40$ & $6: 50$ & $7 \& 17$ & $7: 30 \& 18$ & $9 \& 20$ & 9:40 \\
\hline & Solar fraction (\%) & 85 & 86 & 86 & 88 & 90 & 77 & 77 & 76 & 78 & 80 \\
\hline & Solar fraction change (\%) & 0 & 0 & 0 & 3 & 5 & 0 & 0 & 0 & 3 & 4 \\
\hline
\end{tabular}

Note: * North (N), Northeast (NE), Northwest (NW), East (E), West (W), Southeast (SE), Southwest (SW), South (S); ** Adiabatic (AD)

The maximum solar fraction (98\%) was reached with the use of solar collectors in the upper extreme energy efficiency class, i.e. solar collectors with a 67\% solar energy collection efficiency. Conversely, the use of lower extreme tilt angles (vertical position) and dust accumulation levels (64\% transmittance reduction) in solar collectors promoted a considerable decline in the solar fraction of SWHs, with a minimum of $1 \%$ for thermosiphon SWHs with $64 \%$ dust accumulation. User behaviour also showed a significant influence on the solar fraction of SWHs; for example, lowerand upper-extreme water consumption patterns (77 and 233 litres day-1, respectively) were associated with an increase and decrease of $20 \%$ in the solar fraction of thermosiphon SWHs in relation to the base scenario. By decreasing the 
electric heating element set-point temperature from 60 to $50^{\circ} \mathrm{C}$, the solar fraction of thermosiphon SWHs increased by 20\%; nonetheless, this practice reduces the compliance level with Legionella spp. control temperatures (Table 5).

Table 5. Absolute and relative change in temperature compliance levels for Legionella spp. control.

\begin{tabular}{|c|c|c|c|c|c|c|c|c|c|c|c|}
\hline \multirow{2}{*}{\multicolumn{2}{|c|}{$\begin{array}{c}\text { System type } \\
\text { Energy efficiency class }\end{array}$}} & \multicolumn{5}{|c|}{ Split with stratified vertical tank } & \multicolumn{5}{|c|}{ Thermosiphon with mixed horizontal tank } \\
\hline & & $\mathbf{L E}$ & LS & $\mathbf{S}$ & US & UE & LE & LS & $\mathbf{S}$ & US & UE \\
\hline \multirow{18}{*}{ 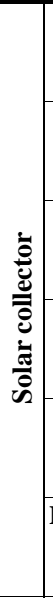 } & Efficiency (\%) & 39 & 43 & 56 & 64 & 67 & 39 & 43 & 56 & 64 & 67 \\
\hline & Compliance (\%) & 67 & 72 & 94 & 94 & 95 & 61 & 66 & 95 & 96 & 97 \\
\hline & Compliance change (\%) & -7 & 0 & 31 & 31 & 32 & -8 & 0 & 44 & 45 & 47 \\
\hline & Area $\left(\mathrm{m}^{2}\right)$ & 1.8 & 2.8 & 5.6 & 9 & 10.4 & 1.8 & 2.8 & 5.6 & 9 & 10.4 \\
\hline & Compliance (\%) & 17 & 41 & 72 & 83 & 85 & 27 & 41 & 66 & 77 & 79 \\
\hline & Compliance change (\%) & -76 & -43 & 0 & 15 & 18 & -59 & -38 & 0 & 17 & 20 \\
\hline & Direction/orientation* & $\mathrm{S}$ & SE/SW & $\mathrm{E} / \mathrm{W}$ & NE/NW & $\mathrm{N}$ & $\mathrm{S}$ & SE/SW & $\mathrm{E} / \mathrm{W}$ & NE/NW & $\mathrm{N}$ \\
\hline & Compliance (\%) & 28 & 36 & 50 & 67 & 72 & 28 & 33 & 51 & 62 & 66 \\
\hline & Compliance change (\%) & -61 & -51 & -31 & -7 & 0 & -57 & -50 & -22 & -6 & 0 \\
\hline & Tilt angle & $90^{\circ}$ & $0^{\circ}$ & $13.7^{\circ}$ & $47.4^{\circ}$ & $27.4^{\circ}$ & $90^{\circ}$ & $0^{\circ}$ & $13.7^{\circ}$ & $47.4^{\circ}$ & $27.4^{\circ}$ \\
\hline & Compliance (\%) & 9 & 51 & 64 & 66 & 72 & 10 & - & 62 & 56 & 66 \\
\hline & Compliance change (\%) & -88 & -30 & -11 & -9 & 0 & -85 & - & -6 & -16 & 0 \\
\hline & Shadowing direction* & $\mathrm{N}$ & $\mathrm{NE} / \mathrm{NW}$ & $\mathrm{E} / \mathrm{W}$ & $\mathrm{S} / \mathrm{SE} / \mathrm{SW}$ & None & $\mathrm{N}$ & $\mathrm{NE} / \mathrm{NW}$ & $\mathrm{E} / \mathrm{W}$ & S/SE/SW & None \\
\hline & Compliance (\%) & 49 & 55 & 69 & 72 & 72 & 56 & 55 & 63 & 66 & 66 \\
\hline & Compliance change (\%) & -31 & -24 & -4 & 0 & 0 & -16 & -18 & -5 & -1 & 0 \\
\hline & Dust radiation decline (\%) & 64 & 47 & 5 & 2.7 & 0 & 64 & 47 & 5 & 2.7 & 0 \\
\hline & Compliance (\%) & 0 & 7 & 72 & 74 & 76 & 1 & 13 & 66 & 68 & 72 \\
\hline & Compliance change (\%) & -100 & -90 & 0 & 3 & 6 & -98 & -81 & 0 & 3 & 8 \\
\hline \multirow{9}{*}{ 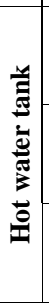 } & Volume (litres) & 90 & 250 & 315 & 450 & 630 & 90 & 250 & 315 & 450 & 630 \\
\hline & Compliance (\%) & 69 & 74 & 72 & 67 & 55 & 56 & 67 & 66 & 65 & 62 \\
\hline & Compliance change (\%) & -4 & 3 & 0 & -7 & -24 & -15 & 1 & 0 & -2 & -6 \\
\hline & Heat loss (W/K) & 3.08 & 1.32 & 0.75 & 0.47 & 0.18 & 3.08 & 1.32 & 0.75 & 0.47 & 0.18 \\
\hline & Compliance (\%) & 32 & 61 & 72 & 78 & 84 & 47 & 61 & 66 & 69 & 72 \\
\hline & Compliance change (\%) & -56 & -15 & 0 & 8 & 17 & -29 & -8 & 0 & 5 & 9 \\
\hline & Cold water connection $\left({ }^{\circ} \mathrm{C}\right)$ & $14-21$ & $16-23$ & $20-27$ & $24-31$ & $26-33$ & $14-21$ & $16-23$ & $20-27$ & $24-31$ & $26-33$ \\
\hline & Compliance (\%) & 68 & 70 & 72 & 76 & 77 & 59 & 61 & 66 & 72 & 76 \\
\hline & Compliance change (\%) & -6 & -3 & 0 & 5 & 7 & -11 & -8 & 0 & 9 & 14 \\
\hline \multirow{6}{*}{ 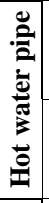 } & Insulation (R-value) & 0 & 0.2 & 0.3 & 1 & $\mathrm{AD}^{* *}$ & 0 & 0.2 & 0.3 & 1 & $\mathrm{AD} * *$ \\
\hline & Compliance (\%) & 68 & 72 & 72 & 72 & 73 & 64 & 66 & 66 & 66 & 70 \\
\hline & Compliance change (\%) & -6 & 0 & 0 & 0 & 2 & -4 & 0 & 0 & -1 & 5 \\
\hline & Length (metres) & 18 & 15 & 9 & 5 & 0 & 18 & 15 & 9 & 5 & 0 \\
\hline & Compliance (\%) & 72 & 72 & 72 & 73 & 73 & 63 & 65 & 66 & 66 & 69 \\
\hline & Compliance change (\%) & 0 & 0 & 0 & 1 & 1 & -5 & -2 & 0 & 0 & 5 \\
\hline \multirow{12}{*}{ 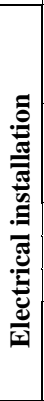 } & Element power (kW) & 5.5 & 4.8 & 3 & 1.5 & 0.9 & 5.5 & 4.8 & 3 & 1.5 & 0.9 \\
\hline & Compliance (\%) & 71 & 72 & 72 & 73 & 72 & 66 & 66 & 66 & 65 & 64 \\
\hline & Compliance change (\%) & -1 & -1 & 0 & 1 & 0 & 0 & 0 & 0 & -2 & -3 \\
\hline & Element tank height (\%) & 0 & 25 & 50 & 75 & 100 & - & - & - & - & - \\
\hline & Compliance (\%) & 87 & 74 & 72 & 70 & 69 & - & - & - & - & - \\
\hline & Compliance change (\%) & 21 & 3 & 0 & -3 & -4 & - & - & - & - & - \\
\hline & Set-point temperature $\left({ }^{\circ} \mathrm{C}\right)$ & 80 & 74 & 60 & 53 & 50 & 80 & 74 & 60 & 53 & 50 \\
\hline & Compliance (\%) & 100 & 100 & 72 & 66 & 65 & 100 & 100 & 66 & 55 & 54 \\
\hline & Compliance change (\%) & 39 & 39 & 0 & -8 & -10 & 52 & 52 & 0 & -17 & -18 \\
\hline & Tariff power interruption (h) & 0 & 2 & 3 & 6 & 16 & 0 & 2 & 3 & 6 & 16 \\
\hline & Compliance (\%) & 72 & 72 & 72 & 72 & 66 & 66 & 66 & 66 & 64 & 57 \\
\hline & Compliance change (\%) & 0 & 0 & 0 & -1 & -9 & 0 & 0 & 0 & -4 & -14 \\
\hline \multirow{9}{*}{ 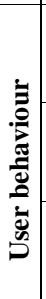 } & End-use temperature $\left({ }^{\circ} \mathrm{C}\right)$ & 50 & 47 & 40 & 35 & 33 & 50 & 47 & 40 & 35 & 33 \\
\hline & Compliance (\%) & 65 & 67 & 72 & 77 & 78 & 57 & 59 & 66 & 74 & 77 \\
\hline & Compliance change (\%) & -10 & -7 & 0 & 6 & 8 & -14 & -11 & 0 & 12 & 16 \\
\hline & Water demand (litres/day) & 233 & 210 & 155 & 100 & 77 & 233 & 210 & 155 & 100 & 77 \\
\hline & Compliance (\%) & 65 & 67 & 72 & 78 & 80 & 59 & 60 & 66 & 76 & 82 \\
\hline & Compliance change (\%) & -10 & -7 & 0 & 8 & 11 & -11 & -9 & 0 & 15 & 24 \\
\hline & Time of peak use hour (24h) & $6: 50$ & $7 \& 17$ & $7: 30 \& 18$ & 9\&20 & $9: 40$ & $6: 50$ & $7 \& 17$ & $7: 30 \& 18$ & $9 \& 20$ & $9: 40$ \\
\hline & Compliance (\%) & 73 & 72 & 72 & 71 & 69 & 80 & 65 & 66 & 66 & 79 \\
\hline & Compliance change (\%) & 1 & -1 & 0 & -1 & -4 & 21 & -2 & 0 & 0 & 20 \\
\hline
\end{tabular}

Note: * North (N), Northeast (NE), Northwest (NW), East (E), West (W), Southeast (SE), Southwest (SW), South (S); ** Adiabatic (AD)

The compliance levels with the Legionella spp. control temperature $\left(60{ }^{\circ} \mathrm{C}\right)$ at the storage tank outlet (top hot water layer) was mainly influenced by the electric heating element set-point temperature. A decrease of this parameter from 60 to $50{ }^{\circ} \mathrm{C}$ showed a reduction of $10 \%$ and $18 \%$ in compliance levels of split and thermosiphon SWHs, respectively; whereas, the increase of this parameter to $74^{\circ} \mathrm{C}$ promoted the full compliance of all SWHs with control temperatures. 
Despite the inhibition of Legionella spp. for temperatures equal or over $60{ }^{\circ} \mathrm{C}$, such bacteria group may persist after heat shocks $\left(70^{\circ} \mathrm{C}\right.$ for 30 minutes).

Results indicate that the characteristics and conditions of solar collectors are critical to achieve low electricity consumption at high compliance levels with Legionella spp. control temperatures. For instance, the use of collectors from the upper-extreme efficiency class showed compliance levels of 95\% and 97\% for split and thermosiphon SWHs, respectively. Conversely, lower-extreme efficiency levels for dust accumulation (64\%) and position (vertical) of solar collectors showed the poorest levels of compliance with control temperatures, i.e.0-1\% and 9-10\%, respectively.

The comparison among the different parameter groups (i.e. technical specifications, site specific conditions, and user behaviour) showed no significant variation for the total annual electricity consumption (null-hypothesis probability (p) $>0.70$ ), solar fraction ( $\mathrm{p}>0.25$ ), peak-hour electricity consumption ( $\mathrm{p}>0.90$ ), and Legionella spp. control temperature compliance ( $>0.25)$. The energy intensity was not tested as it is a direct function of the annual electricity consumption; while, results for the end-use temperature were not compared due to the full compliance of studied scenarios with this indicator. Fig. 2 illustrates the Coefficient of Variation (CV) of assessed parameters for key performance indicators.

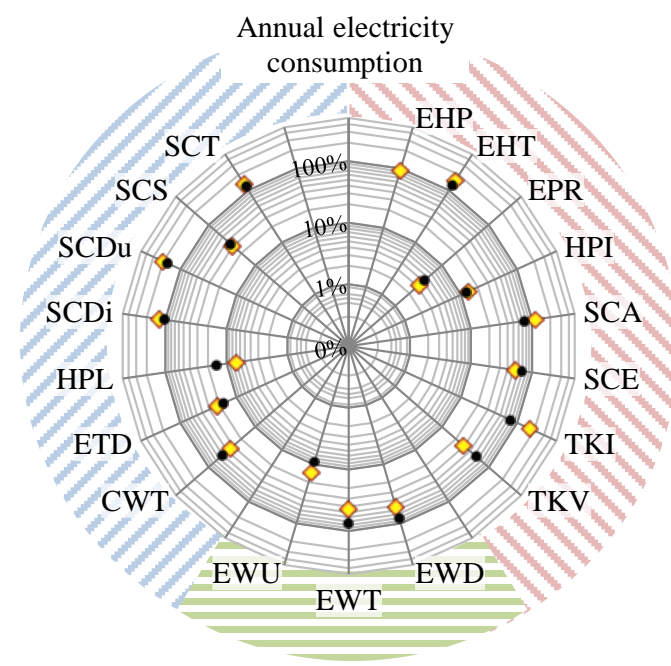

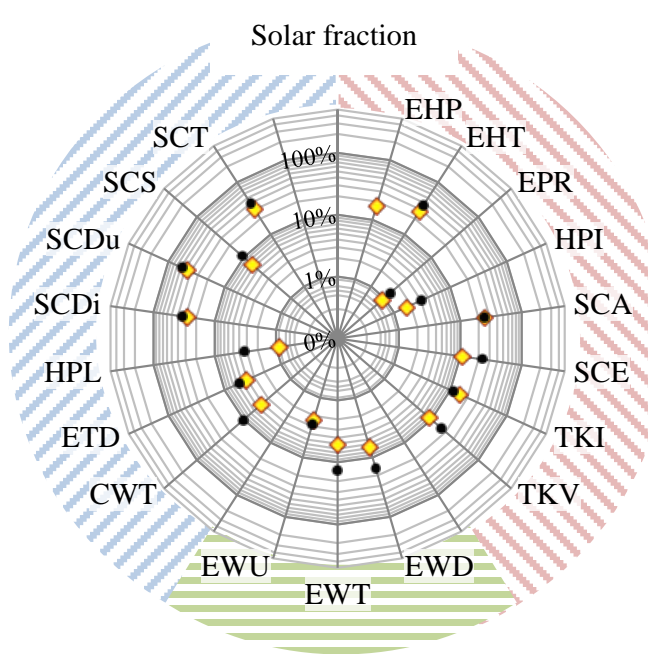

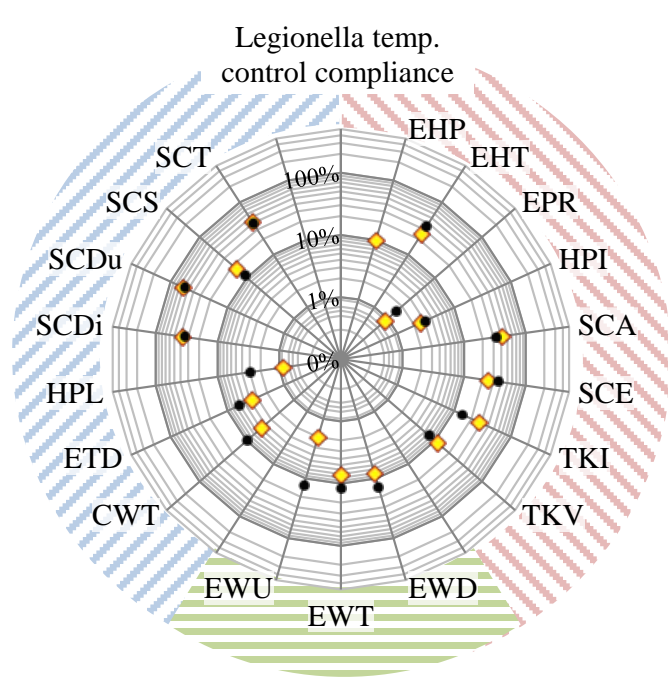

Site-specific conditions

Technical specifications

User behaviour $\diamond$ Split SWHs

- Thermosiphon SWHs

Fig. 2. Coefficient of variation of energy performance and service of level indicators.

Note: Solar water heaters (SWHs), Electric back-up heating position (EHP), Electric back-up heating temperature range (EHT), Electric back-up heating power rate (EPR), Hot water pipe insulation (HPI), Solar collector area (SCA), Solar collector efficiency (SCE), Tank heat loss (TKI), Tank volume (TKV), End-use water demand (EWD), End-use water temperature (EWT), End-use 
water time-of-use (EWU), Cold water temperature (CWT), Electricity tariff time-of-use (ETD), Hot water pipe length (HPL), Solar collector direction (SCDi), Solar collector dust accumulation (SCDu), Solar collector shadowing (SCS), and (Solar collector tilt angle (SCT).

The variability of the annual electricity consumption expressed in CV of split and thermosiphon systems were directly correlated with a Coefficient of Determination $\left(\mathrm{R}^{2}\right)$ equal to 0.84 , which indicates a similar influence of parameters on both system types. Nonetheless, split systems were more sensitive to oscillations on the parameters with the largest impact on both systems. For example, the respective coefficients of variation of thermosiphon and split systems related to the annual electricity consumption were $170 \%$ and $202 \%$ for solar collector dust accumulation, $77 \%$ and $167 \%$ for tank heat loss, $127 \%$ and $158 \%$ for electric back-up heating temperature range, $120 \%$ and $134 \%$ for solar collector tilt angle, $105 \%$ and $125 \%$ for solar collection direction, and $75 \%$ and $114 \%$ for solar collector area. On the other hand, thermosiphon systems were more influenced than split systems by parameters with intermediate influence on SWHs, for which the respective CVs of split and thermosiphon systems were 52\% and 79\% for end-use water demand, 44\% and 75\% for end-use water temperature, 54\% and 69\% for solar collector efficiency, 29\% and 54\% for tank volume, and 35\% and $51 \%$ for cold water temperature. The remaining parameters had less than 35\% variability for both split and thermosiphon systems. The six largest variabilities in the sensitivity analysis of annual electricity consumption were associated with three site-specific parameters and three technical specifications categories. Thus, in order to attain energy efficiency through the use of SWHs, site-specific conditions must be considered along with technical specifications. Moreover, user behaviour also promoted a significant variation in the electricity consumption of SWHs.

The variability of the solar fraction indicator followed the same trend as the annual electricity consumption, with a direct correlation of $84 \%\left(\mathrm{R}^{2}=0.82\right)$. This similarity is related to the inverse correlation between these two indicators. For instance, their correlation was equal to $95 \%$ for thermosiphon systems, and $91 \%$ for split systems. The comparison of the solar fraction variability between split and thermosiphon systems revealed a larger sensitivity of the latter system type due to changes in the input parameter classes. Not only did split systems have a consistently higher solar fraction than thermosiphon systems (9\% higher on average), but also their solar fraction was more resilient to modifications in technical specifications, site-specific conditions and user behaviour patterns. The solar fraction changeability of split systems was only higher than that for thermosiphon systems for the hot water tank heat loss parameter. For this parameter, the solar energy gains of split systems were consistently $10 \%$ above thermosiphon systems for the standard, upper-standard and upper-extreme classes; albeit, for the lower-extreme class, this difference was reduced to only $1 \%$, for thermosiphon systems were not as prone as split systems to heat losses due to their lower average hot water temperature within hot water tanks.

The peak-hour electricity consumption measure in absolute terms $\left(\mathrm{kWh}^{-} \mathrm{year}{ }^{-1}\right)$ was the most variable indicator. This indicator was particularly influenced by changes in the input parameters of split systems, reaching the single highest variability among parameters ( $\mathrm{CV}=973 \%)$ for the electricity back-up heating position parameter. This extremely high oscillation is associated with an increase in auxiliary heating events when the electric heating element is positioned between the lower half and the bottom of hot water tanks; under these conditions, the peak-hour electricity consumption increased from approximately 17 to a maximum of $381 \mathrm{kWh}^{\cdot} \mathrm{year}^{-1}$. On the contrary, the position of elements had neutral influence on peak-hour electricity consumption of thermosiphon systems (approximately $91 \mathrm{kWh}^{\cdot} \mathrm{year}^{-1} \mathrm{on}^{\mathrm{n}}$ average) due to the mixing of hot water into horizontal tanks. Among parameters with impact on both system types, the solar collector direction promoted the largest fluctuation of the peak-hour electricity consumption, i.e. CV=254\% for thermosiphon systems and $\mathrm{CV}=567 \%$ for split systems. The solar collector tilt angle also had a considerable effect on the peak-hour electricity of thermosiphon $(\mathrm{CV}=118 \%)$ and split $(\mathrm{CV}=370 \%)$ systems. The electric back-up heating range (hot water tank set-point temperature) showed a significant modification of the peak-hour electricity consumption as well, for which the coefficient of variation of thermosiphon and split systems were 142 and 159\%, respectively. The dust accumulation effect on collectors was associated with a significant expansion of peak-hour electricity consumption 
of split systems (up to $174 \mathrm{kWh} \cdot \mathrm{year}^{-1}$ ), and hence it had the third largest influence on the variability of split systems (CV=430\%) with a considerably lower significance for thermosiphon systems (CV=46\%). Electricity tariffs also played a major role in the peak-hour electricity consumption variability of split systems (CV=85\%) and thermosiphon systems ( $\mathrm{CV}=89 \%)$, as they can provide a full diversion of electricity consumption from peak-hours. Other parameters had an overall lower significance on the peak-hour electricity variability than the aforementioned parameters.

The variability of the compliance level with Legionella spp. control temperature showed a significant lower range than the one for annual and peak-hour electricity consumption. Its average was slightly higher for thermosiphon systems (11\%) in relation to split systems (14\%). The parameters with the largest influence on the compliance levels of both system types were associated with solar collectors. For instance, the site specific conditions related to the dust accumulation and tilt angle of collectors had the largest variabilities among all parameters, with respective coefficients of variation of $57 \%$ for thermosiphon and $60 \%$ for split systems, and $39 \%$ for thermosiphon and $42 \%$ for split systems. Moreover, the specification of the area of solar collectors was also linked to a large variability of Legionella spp. control temperatures in relation to other parameters, i.e. $\mathrm{CV}=34 \%$ for thermosiphon systems and $\mathrm{CV}=37 \%$ for split systems. The second most important component of the SWHs in terms of Legionella spp. control temperature compliance was the hot water tank. Its auxiliary electricity heating element setting-point temperature was the major parameter to promote variability in the control temperature ( $\mathrm{CV}=34 \%$ for thermosiphon systems and $\mathrm{CV}=25 \%$ for split systems) followed by the insulation specification ( $C V=14 \%$ for thermosiphon systems and $C V=27 \%$ for split systems) and the tank volume (CV=8\% for thermosiphon systems and 11\% for split systems). The user behaviour also had a significant impact on the control temperature in particular for thermosiphon systems. For instance, the coefficient of variation of the end-use point temperature, time-of-use and demand for thermosiphon systems was 12, 13 and 14\%, respectively; while, for split systems, respective results for the same parameters were equal to 5, 8 and 7\%. The influence of other parameters on this indicator was not as pronounced as the one of the aforementioned parameters.

The multi-parametric sensitivity analysis indicates a predominant influence of parameters related to solar collectors due to their importance for the adequate performance of SWHs. Among these parameters, direction, dust accumulation levels and area of solar collectors were the most influential parameters related to the total energy consumption variability. The reduced influence of user behaviour parameters on the total energy consumption and Legionella spp. control temperature is mainly associated with the adequate design of the standard system, in which the hot water supply is suitable for a range of demand scenarios. Conversely, the impact of user behaviour on peak-hours electricity consumption was significant, revealing the importance of a proactive attitude of households towards daily consumption pattern adjustments to reduce peak consumption. Alternatively, peak-hours electricity consumption can be also eliminated without significant impacts to other performance indicators by adopting off-peak electricity tariffs. Thus, the optimisation of SWHs under a multi-parametric framework encompasses multiple influential parameters, which have to be refined on a case-by-case basis.

\section{Key findings}

The study showed a significant influence of different technical specifications and site-specific conditions on the performance of SWHs considering both energy and service level indicators. A summary of key findings is presented in the following sub-sections.

\subsection{Supply reliability}

SWHs can achieve full compliance with comfort requirements (i.e. continuous hot water supply at end-use points at $40^{\circ} \mathrm{C}$ ) under variable conditions, albeit there may be some time periods where the temperature of systems may not be 
adequate for Legionella spp. growth control (i.e. continuous hot water storage measured at the outlet of the storage tank at $60{ }^{\circ} \mathrm{C}$ or more) in particular during cold months.

Care should be practiced in the interpretation of results related to Legionella spp. control temperature. As described by Kruse et al. [28], the susceptibility of residential hot water system to Legionella spp. proliferation cannot be easily linked to clinical cases of legionellosis. Nonetheless, Mercante and Winchel [27] indicate that the number of legionellosis are likely underestimated due to limitations in diagnostic and detection methods, and that this disease may pose mortality risk among at-risk populations (e.g. immunosuppressed and elderly populations).

\subsection{Split versus thermosiphon systems}

Split systems have an overall better performance than thermosiphon systems under standard specification and operation conditions. The total annual electricity consumption of split systems was $7 \%$ lower than the one for thermosiphon systems, with a reduction in peak-hour consumption of $20 \%$ and a $6 \%$ higher compliance level with Legionella spp. control temperatures. Nonetheless, thermosiphon systems were more energy efficient during summer, when they reached almost $100 \%$ solar fraction.

Thermosiphon systems had a better performance under low water demand and temperature patterns, while split systems performed better under high water demand and temperature patterns. For instance, by comparison with the adopted standard user behaviour (155 litres household $^{-1} \cdot$ day $^{-1}$ at $40^{\circ} \mathrm{C}$ ), the upper-extreme classes for temperature (155 litres $\cdot$ household ${ }^{-1} \cdot \operatorname{day}^{-1}$ at $\left.50{ }^{\circ} \mathrm{C}\right)$ and water demand $\left(233\right.$ litres $\cdot$ household ${ }^{-1} \cdot$ day $^{-1}$ at $\left.40^{\circ} \mathrm{C}\right)$ promoted an increase in the electricity consumption of $123 \%$ in thermosiphon systems; in split systems, the same parameter classes were associated with an expansion in the electricity consumption of $68 \%$ and $80 \%$, respectively. On the other hand, the lower-extreme classes for temperature $\left(155\right.$ litres household $^{-1} \cdot$ day $^{-1}$ at $\left.33^{\circ} \mathrm{C}\right)$ and water demand $\left(77\right.$ litres $\cdot$ household $^{-1} \cdot \mathrm{day}^{-1}$ at $\left.40^{\circ} \mathrm{C}\right)$ were associated with respective electricity saving potentials of $39 \%$ and $52 \%$ in split systems, and $65 \%$ and $76 \%$ in thermosiphon systems.

The hot water tank volume had a higher impact on the total annual electricity consumption of thermosiphon systems (CV=54\%) than split systems (CV=29\%). Conversely, the peak-hour electricity consumption was more influenced by the tank volume in split systems ( $C V=51 \%)$ than thermosiphon systems ( $C V=21 \%)$. For a standard pipe length from the hot water tank to the supply point of 9 meters and standard to upper-extreme levels of pipe insulation $\left(0.75-3.08 \mathrm{~W} \cdot \mathrm{K}^{-1}\right)$, electricity saving potentials varied between $14 \%$ and $45 \%$ for split systems and between $35 \%$ and $50 \%$ for thermosiphon systems. Split systems are more sensitive to solar collector area variations than thermosiphon systems. Hence, in comparison with thermosiphon systems, the electricity consumption of split systems was $18 \%$ higher and $25 \%$ lower considering the use of lower-extreme $\left(1.8 \mathrm{~m}^{2}\right)$ and upper-extreme $\left(10.4 \mathrm{~m}^{2}\right)$ solar collector areas.

\subsection{Solar collectors}

Despite the widespread use of SWHs with lower-standard efficiency solar collectors, there is a significant potential for electricity savings by using more efficient collectors. For instance, the use of more efficient solar collectors showed electricity saving potentials from 65 to $71 \%$ for split systems and from $83 \%$ to $91 \%$ for thermosiphon systems.

The performance of SWHs can decline considerably for solar collector orientations other than north facing, e.g. east and west orientation of collectors may cause a reduction in the electricity saving potential of 80-83\% and a decline in Legionella spp. control temperature to compliance levels of 49-53\%. Collectors facing south cardinal and inter-cardinal directions not only lead to a significant decline in the energy performance of SWHs, but also to critical levels of compliance with Legionella spp. control temperature (28-36\%). The performance of SWHs was also highly influenced by the inclination (tilt angle) of solar collectors. For instance, in relation to the latitude angle, the vertical installation of 
collectors on the façade of buildings may decrease the solar fraction and the Legionella spp. control temperature of SWHs by $56-63 \%$.

In relation to clean solar collectors, the standard level of dust accumulation with a $5 \%$ transmittance reduction on the glass of solar collectors promoted an increase in the electricity consumption of SWHs equal to 20-24\%. For extreme levels of dust accumulation (i.e. 64\% transmittance reduction), the annual electricity consumption of SWHs increased by 5 to 6 -fold. North shadowing has the potential to reduce Legionella spp. control temperatures and energy saving performance of SWHs by $11-23 \%$ and $72-75 \%$, respectively.

\subsection{Interconnected systems}

The electricity connection influenced considerably the performance of SWHs. Peak-hour electricity consumption can be diverted to shoulder and off-peak-hours by using controlled tariffs without any impact to the Legionella spp. control temperature. Moreover, night off-peak tariffs can be used to achieve full diversion of peak-hour and shoulder hour electricity consumption, yet with a decline in the compliance level with Legionella spp. control temperatures of 6-9\%. The cold water connection temperature has a direct influence on the performance SWHs. For instance, the electricity savings potential can be improved by $38 \%$ to $56 \%$ or deteriorated by $52 \%$ to $74 \%$ with a respective increase or decrease of $6{ }^{\circ} \mathrm{C}$ on the average cold water supply temperature.

\section{Market applications}

Taking into account the conditions assessed in the present study, the energy efficiency and the level of service provided by SWHs could be enhanced through the adoption of the following practices into the SWHs market:

- $\quad$ Split systems is the preferred option for SWHs with medium to large solar collector areas $\left(>5.5 \mathrm{~m}^{2}\right)$ in households, while thermosiphon systems have a better performance if solar collectors are undersized.

- The energy performance of split SWHs can be further improved by adopting photovoltaic powered pumps to circulate the heating fluid (water) in collectors. This initiative has the potential to promote electricity saving of $15 \%$ on average, and can eliminate any disadvantage of split system in comparison with thermosiphon systems under low water demand and temperature patterns or for undersized solar collectors.

- Thermosiphon systems have a lesser potential to reduce electricity consumption during peak-hours than split systems, and hence they should be a secondary system type adopted into policies aimed at a reduction of peak-hour electricity consumption.

- The service performance of SWHs has to be analysed based not only on the comfort level, but also on the capacity to maintain high hot water storage temperatures to prevent Legionella spp. growth. This information has to be shared among all stakeholder groups, including but not limited to users who may deem the performance of systems being satisfactory taking into account the comfort level only. As a result, practices such as the manual activation and inactivation of electric heating elements on power switches by users based on their comfort level should be eliminated to prevent public health risks.

- The set-point temperature of storage tanks can be increased during cold months in order to maintain adequate levels of protection against Legionella spp. proliferation. Alternatively, other systems can be used for disinfection, e.g. ultraviolet disinfection systems coupled to the solar collector and/or water supply circulation loops.

- The adoption of SWHs with efficient solar collectors (standard, upper-standard or upper-extreme classes) will usually be required should higher levels of Legionella spp. control and solar fraction be required. 
- The installation of solar collectors toward orientations other than north cardinal and inter-cardinal directions must be performed taking into account measures to improve the compliance with Legionella spp. control temperatures, including but not limited to: adoption of efficient collectors, increase in the hot water tank set-point temperature, alternative disinfection systems, etc.

- $\quad$ The installation of SWHs with solar collectors oriented to east and west directions must be undertaken under a thorough system performance assessment framework considering site-specific conditions prior to commissioning in order to prevent poor energy efficiency and service levels.

- Considering the significant influence of the cold water temperature on the performance of SWHs, heat recovery from drain water can be implemented to improve the energy efficiency of SWHs by increasing the cold water temperature. An example of such systems is described by Tanha et al. [17].

- The operational performance of SWHs must be assessed periodically or continuously, as variable site-specific conditions (e.g. dust accumulation, trees shadowing, etc.) may have a significant impact on Legionella spp. control temperatures and energy performance levels of systems, while maintaining adequate levels of hot water supply at the end-use points with the use of auxiliary heating systems (electric resistance elements). Hence, the “install-and-forget” approach is not suitable for SWHs. This limitation can be overcome with the adoption of automated metering technologies for energy consumption and temperature compliance levels of SWHs with remote data transmission to relevant stakeholders (e.g. households, building administrators, energy retailors, water utilities, health departments and/or energy departments). Depending on the automated metering technology used, a two-way communication with SWHs can be deployed, enabling the remote adjustment of operational parameters (e.g. auxiliary heating temperature set-point, time-of-use of electricity at premises with a single electricity meter and no power interruption during peak-hours, etc.). The economic feasibility of such technologies is increasing with an expansion of microcontrollers and sensors availability, as well as cloud-base big data analytics platforms and the Internet of Things (IoT).

- If a decline in the performance of SWHs is identified or higher levels of performance are required, a mitigation/remediation process can be implemented, which may vary from simple measures (e.g. cleaning up solar collectors) to more complex ones (e.g. installing new components such as new insulation of pipes, frames to position collectors to north orientations at latitude tilt angles, etc.); however, complex initiatives may require government subsidies. A change in the user behaviour may also have a significant impact on the performance of SWHs. For instance, e.g. shifting from an early morning to a late morning water consumption peak may give rise to electricity savings of $14 \%$ in thermosiphon and $29 \%$ in split systems.

- If night off-peak tariffs are used to increase the solar fraction of SWHs and prevent electricity consumption during peak and shoulder hours, Legionella spp. control temperature compliance levels must be assessed and accompanied by additional control measures should it be required. 


\section{Conclusions}

The current study examined the influence of different technical specifications and site-specific conditions on the performance of SWHs in residential buildings in Brisbane, Australia. The study was undertaken by employing sensitivity analysis using computational models calibrated with experimental measurements.

Results indicate that the annual total and peak-hour electricity consumption and the Legionella spp. control temperature of SWHs varied significantly (CV>10\%) with the adoption of different confidence intervals for technical specification parameters, site-specific conditions and user behaviour. Dust accumulation level on collectors is a key site-specific parameter for the energy performance and level of service of SWHs, outplaying several technical specification parameters (e.g. solar collector area and efficiency). Therefore, the development of energy efficiency policies on thermal solar energy use must contemplate directions and best practices on the operation of systems considering site-specific conditions, e.g. frequency of solar collector dust removal events in order to maintain the intended levels of energy efficiency and service as per commissioning process.

In this context, performance analysis frameworks for SWHs have to be based not only on technical specification parameters and energy efficiency indicators, but also they must take into account site-specific and user behaviour parameters and level of service indicators.

\section{Acknowledgements}

The authors acknowledge the Campus Life team from the Nathan campus of Griffith University, in particular Mr. Geoff Smith (Campus Life Maintenance Supervisor), for assisting with the technical aspects of the field experiment. Also, the authors would like to thank the PhD candidate Mr. Domagoj Leskarac at Griffith University for his help in obtaining data from the weather station adjacent to the experiment. 


\section{References}

[1] H. Turton, Greenhouse gas emissions in industrialised countries Where does Australia stand ?, Discussion Paper Number 66, 2004.

[2] T. Yusaf, S. Goh, J. A. Borserio, Potential of renewable energy alternatives in Australia, Renew. Sustain. Energy Rev. 15 (2011) 2214-2221, http://dx.doi.org/10.1016/j.rser.2011.01.018.

[3] A. Baniyounesa, G. Liu, M.G. Rasul, M.M.K. Khan, Review on Renewable Energy Potential in Australian Subtropical Region (Central and North Queensland), Adv. Mater. Res. 347-353 (2012) 3846-3855, http://dx.doi.org/10.4028/www.scientific.net/AMR.347-353.3846.

[4] K. Lovegrove, M. Dennis, Solar thermal energy systems in Australia, Int. J. Environ. Stud. 63 (2006) 791-802, http://dx.doi.org/10.1080/00207230601047156.

[5] A. Bahadori, C. Nwaoha, A review on solar energy utilisation in Australia, Renew. Sustain. Energy Rev. 18 (2013) 1-5, http://dx.doi.org/10.1016/j.rser.2012.10.003.

[6] Commonwealth of Australia, Media release: Certainty and growth for renewable energy, 2015. http://www.environment.gov.au/minister/hunt/2015/pubs/mr20150623.pdf (accessed 01/Sep/2017).

[7] Clean Energy Council, A Bipartisan Renewable Energy Target: The Huge Opportunities for Australia, 2015. https://www.cleanenergycouncil.org.au/policy-advocacy/renewable-energy-target.html (Accessed 01/Sep/2017).

[8] G. Baverstock, A. Gaynor, Fifty Years of Solar Energy in Australia and New Zealand: A history of the Australian and New Zealand Solar Energy Society, ANZSES, 2010.

[9] D. Mills, Assessing solar hot water system performance with GIS, Sol. Energy 76 (2004) 153-157, http://dx.doi.org/10.1016/j.solener.2003.07.029.

[10] AS/NZS 4234:2008, Heated water systems-Calculation of energy consumption, 2001.

[11] A. Allouhi, A. Jamil, T. Kousksou, T. El Rhafiki, Y. Mourad, Y. Zeraouli, Solar domestic heating water systems in Morocco: An energy analysis, Energy Convers. Manag. $92 \quad$ (2015) 105-113, http://dx.doi.org/10.1016/j.enconman.2014.12.055.

[12] J.L. Duomarco, Figure of merit for solar domestic hot water systems, Sol. Energy 111 (2015) 151-156, http://dx.doi.org/10.1016/j.solener.2014.10.023.

[13] E. Halawa, K.C. Chang, M. Yoshinaga, Thermal performance evaluation of solar water heating systems in Australia, Taiwan and Japan - A comparative review, Renew. Energy 83 (2015) 1279-1286, http://dx.doi.org/10.1016/j.renene.2015.04.023.

[14] R. Li, Y. Dai, R. Wang, Experimental investigation and simulation analysis of the thermal performance of a balcony wall integrated solar water heating unit, Renew. Energy $75 \quad$ (2015) 115-122, http://dx.doi.org/10.1016/j.renene.2014.09.023.

[15] C. Yan, S. Wang, Z. Ma, W. Shi, A simplified method for optimal design of solar water heating systems based on life-cycle energy analysis, Renew. Energy $74 \quad$ (2015) 271-278, http://dx.doi.org/10.1016/j.renene.2014.08.021.

[16] A. Saravanan, J.S. Senthilkumaar, S. Jaisankar, Performance assessment in V-trough solar water heater fitted with square and V-cut twisted tape inserts, Appl. Therm. Eng. 102 (2016) 476-486.

[17] K. Tanha, A.S. Fung, R. Kumar, Performance of two domestic solar water heaters with drain water heat recovery units: Simulation and experimental investigation, Appl. Therm. Eng. 90 (2015) 444-459, http://dx.doi.org/10.1016/j.applthermaleng.2015.07.038. 
[18] H.S. Xue, Experimental investigation of a domestic solar water heater with solar collector coupled phase-change energy storage, Renew. Energy $86 \quad$ (2016) 257-261, http://dx.doi.org/10.1016/j.renene.2015.08.017.

[19] C. Lamnatou, J.D. Mondol, D. Chemisana, C. Maurer, Modelling and simulation of Building-Integrated solar thermal systems: Behaviour of the coupled building/system configuration, Renew. Sustain. Energy Rev. 48 (2015) 178-191, http://dx.doi.org/10.1016/j.rser.2015.03.075.

[20] D. Li, G. Liu, S. Liao, Solar potential in urban residential buildings, Sol. Energy 111 (2015) 225-235, http://dx.doi.org/10.1016/j.solener.2014.10.045.

[21] S. Edwards, I. Beausoleil-Morrison, A. Laperrière, Representative hot water draw profiles at high temporal resolution for simulating the performance of solar thermal systems, Sol. Energy 111 (2015) 43-52, http://dx.doi.org/10.1016/j.solener.2014.10.026.

[22] N. Gill, P. Osman, L. Head, M. Voyer, T. Harada, G. Waitt, C. Gibson, Looking beyond installation: Why households struggle to make the most of solar hot water systems, Energy Policy 87 (2015) 83-94, http://dx.doi.org/10.1016/j.enpol.2015.08.038.

[23] W.M. Lin, K.C. Chang, K.M. Chung, Payback period for residential solar water heaters in Taiwan, Renew. Sustain. Energy Rev. 41 (2015) 901-906, http://dx.doi.org/10.1016/j.rser.2014.09.005.

[24] E. Bédard, S. Fey, D. Charron, C. Lalancette, P. Cantin, P. Dolcé, C. Laferrière, E. Déziel, M. Prévost, Temperature diagnostic to identify high risk areas and optimize Legionella pneumophila surveillance in hot water distribution systems, Water Res. 71 (2015) 244-256, http://dx.doi.org/10.1016/j.watres.2015.01.006.

[25] I. Boppe, E. Bédard, C. Taillandier, D. Lecellier, M.-A. Nantel-Gauvin, M. Villion, C. Laferrière, M. Prévost, Investigative approach to improve hot water system hydraulics through temperature monitoring to reduce building environmental quality hazard associated to Legionella, Build. Environ. 108 (2016) 230-239, http://dx.doi.org/10.1016/j.buildenv.2016.08.038.

[26] HSE, Legionnaires’ disease - Part 2: The control of legionella bacteria in hot and cold water systems, 2014.

[27] J.W. Mercante, J.M. Winchell, Current and Emerging Legionella Diagnostics for Laboratory and Outbreak Investigations, Clin. Microbiol. Rev. 28 (2015) 95-133, http://dx.doi.org/10.1128/CMR.00029-14.

[28] E. Kruse, A. Wehner, H. Wisplinghoff, Prevalence and distribution of Legionella spp in potable water systems in Germany, risk factors associated with contamination, and effectiveness of thermal disinfection, Am. J. Infect. Control. 44 (2016) 470-474, http://dx.doi.org/10.1016/j.ajic.2015.10.025. 
Supplementary material to:

\title{
'Residential solar water heaters in Brisbane, Australia: key performance parameters and indicators'
}

\author{
Abel S. Vieira ${ }^{\mathrm{a}}$, Rodney A. Stewart ${ }^{\mathrm{a}}$, Roberto Lamberts ${ }^{\mathrm{b}}$, Cara D. Beal ${ }^{\mathrm{c}}$ \\ a School of Engineering, Griffith University, Gold Coast Campus, Queensland 4222, Australia; ${ }^{b}$ Federal \\ University of Santa Catarina, Department of Civil Engineering, Field of Energy Efficiency in Buildings, \\ Florianópolis, 88040-900, SC, Brazil; ' Smart Water Research Centre and School of Engineering, Griffith \\ University, Gold Coast Campus, Queensland 4222, Australia.
}

Corresponding author:

Professor Rodney Stewart

Griffith School of Engineering, Gold Coast Campus, Griffith University, Parklands Drive, Southport, QLD 4222 Australia;

Phone: +61 755528778

Email: r.stewart@griffith.edu.au

\section{Nomenclature}

A Surface area considered per unit area $-1\left(\mathrm{~m}^{2}\right)$

ASHRAE American Society of Heating, Refrigerating and Air Conditioning Engineers

$\mathrm{c}_{0} \quad$ Optical coefficient (dimensionless)

$\mathrm{c}_{1} \quad$ Linear loss coefficient $(\mathrm{W} \cdot \mathrm{m}-2 \cdot \mathrm{K})$

$\mathrm{C}_{2} \quad$ Quadratic loss coefficient $(\mathrm{W} \cdot \mathrm{m}-2 \cdot \mathrm{K} 2)$

DEWS Department of Energy and Water Supply

$\mathrm{H} \quad$ Thermal efficiency coefficient (dimensionless)

HL Heat loss coefficient $(\mathrm{W} \cdot \mathrm{K}-1)$

Isolar Global incident solar radiation $(\mathrm{W} \cdot \mathrm{m}-2)$

$\mathrm{K} \quad$ Thermal conductivity of the insulation material $(\mathrm{W} \cdot \mathrm{m}-1 \cdot \mathrm{K}-1)$

L Thickness of the insulation material (m)

LE Lower-extreme energy efficiency class

LS Lower-standard energy efficiency class

$\mathrm{R} \quad$ Thermal resistance $(\mathrm{K} \cdot \mathrm{W}-1)$

RMY Representative Meteorological Year

$S \quad$ Standard energy efficiency class (Mean value)

SRCC-US Solar Rating and Certification Corporation of the United States

$\mathrm{T}_{\text {air }} \quad$ Outdoor air temperature $\left(\mathrm{K}^{\circ}\right.$ or $\left.\mathrm{C}^{\circ}\right)$

$\mathrm{T}_{\text {in }} \quad$ Inlet temperature of the working fluid $\left(\mathrm{K}^{\circ}\right.$ or $\left.\mathrm{C}^{\circ}\right)$

UE Upper-extreme energy efficiency class

US Upper-standard energy efficiency class

US-DOE United States Department of Energy

$\mathrm{V}_{95} \quad$ Extreme value at 95\% confidence interval (lower-standard or upper-standard values)

$\mathrm{V}_{99} \quad$ Extreme value at a 99\% confidence interval (lower-extreme or upper-extreme values)

$\mathrm{z}_{95} \quad 95 \%$ confidence interval factor from the Gaussian distribution

$\mathrm{z}_{99} \quad 99 \%$ confidence interval factor from the Gaussian distribution 


\section{S-A. Computational model calibration}

\section{Technique and input parameters}

Computational models were undertaken using the software EnergyPlus 8.6 from the United States Department of Energy (US-DOE). This software is largely used for energy performance analysis in buildings [1-7].

Moreover, EnergyPlus is freely distributed, while most of other software packages with solar energy modelling capabilities have licensing fees, e.g. TRNSYS, SolDesigner, SolarPro, and T*SOL [8].

Theoretical results from models were calibrated and validated by means of comparison of energy performance results with experimental results from a field experiment. The validation process comprised of emulating field conditions based on the technical specifications and weather condition data from the SWH experiment followed by a comparison between experimental and theoretical energy consumption results. Model validation utilised field experiment data collected between the period of 20 September 2014 and 10 October 2014.

The field data related to the weather condition were converted into the required weather file format for models (i.e. EPW files) using the Weather and Statistic Conversion software, which is part of the auxiliary EnergyPlus programs. The conversion was performed for the following parameters: cold water temperature, outside dry bulb air temperature and relative humidity, global solar radiation, and wind speed. All the input parameters were converted to an hourly basis to match the model input schedules. The direct normal radiation and diffuse radiation were estimated by the Weather and Statistic Conversion software using the method described by Perez et al. [9]. Likewise, the dewpoint temperature was estimated using the relative humidity and the barometric pressure for Brisbane based on the method described by the American Society of Heating, Refrigerating and Air Conditioning Engineers (ASHRAE) Handbook of Fundamental [10].

The input parameters used in the validation model are summarised in Table S-A 1. Models were also used to analyse multiple scenarios for different SWH technical specifications, site-specific characteristics and user behaviour patterns in the sensitivity analysis.

Table S-A 1. Input parameters for computational model validation.

\begin{tabular}{|c|c|c|c|}
\hline Parameter & Unit & Value & Source \\
\hline Air temperature and relative humidity & ${ }^{\circ} \mathrm{C}$ & Oscillating & Experimental data \\
\hline Cold water temperature & ${ }^{\circ} \mathrm{C}$ & Oscillating & Experimental data \\
\hline End-use temperature & ${ }^{\circ} \mathrm{C}$ & 36 to 39 & Experimental data \\
\hline $\begin{array}{l}\text { Lower and upper hot water tank } \\
\text { temperature thresholds }\end{array}$ & ${ }^{\circ} \mathrm{C}$ & 52 and 60 & Technical specification \\
\hline Storage tank shape & - & Horizontal cylinder & Experimental data \\
\hline $\begin{array}{l}\text { Number of water stratification nodes in the } \\
\text { storage tank }\end{array}$ & - & 12 & $\begin{array}{l}\text { Maximum model } \\
\text { capability }\end{array}$ \\
\hline Storage tank volume & litres & 233 & Experimental data \\
\hline Storage tank height of cold water inlet point & metre & 0.080 & Experimental data \\
\hline $\begin{array}{l}\text { Storage tank height of hot water outlet } \\
\text { point }\end{array}$ & metre & 0.330 & Experimental data \\
\hline $\begin{array}{l}\text { Storage tank height of hot water inlet point } \\
\text { from collectors }\end{array}$ & metre & 0.200 & Experimental data \\
\hline Height of auxiliary electric heating element & metre & 0.205 & Experimental data \\
\hline Power rate of the auxiliary electric heating & W & 1800 & Technical specification \\
\hline
\end{tabular}




\begin{tabular}{|c|c|c|c|}
\hline $\begin{array}{l}\text { Heat loss coefficient - Upper } 2 / 3 \text { of storage } \\
\text { tank }\end{array}$ & $\mathrm{W} \cdot \mathrm{K}^{-1}$ & $\begin{array}{l}0.45 \text { (average } \\
\text { polyurethane insulation } \\
\text { of } 55 \mathrm{~mm} \text { at thermal } \\
\text { conductivity of } 0.025 \\
\mathrm{~W} \cdot \mathrm{m}^{-1} \cdot \mathrm{K}^{-1} \text { ) }\end{array}$ & Experimental data \\
\hline $\begin{array}{l}\text { Heat loss coefficient - Lower } 1 / 3 \text { of storage } \\
\text { tank }\end{array}$ & $\mathrm{W} \cdot \mathrm{K}^{-1}$ & $\begin{array}{l}2.08 \text { (average } \\
\text { polyurethane insulation } \\
\text { of } 12 \mathrm{~mm} \text { at thermal } \\
\text { conductivity of } 0.025 \\
\mathrm{~W} \cdot \mathrm{m}^{-1} \cdot \mathrm{K}^{-1} \text { ) }\end{array}$ & Experimental data \\
\hline Pipe heat loss coefficient* & $\mathrm{kW} \cdot \mathrm{K}^{-1}$ & $\begin{array}{l}130 \text { (copper pipe with } \\
3 \mathrm{~mm} \text { wall at thermal } \\
\text { conductivity of } 390 \mathrm{~W} \cdot \mathrm{m}^{-} \\
{ }^{1} \cdot \mathrm{K}^{-1} \text { ) }\end{array}$ & Experimental data \\
\hline
\end{tabular}

Note: * The copper pipe lengths without insulation were installed in the collector inlet branch (2.1 m pipe from the tank bottom connection to the solar collectors inlet connection) and the collector outlet branch ( $0.4 \mathrm{~m}$ pipe from the solar collector outlet connection to the tank middle connection) as per the local SWHs retailers/installers practice.

\subsection{Field experiment}

Field experiments were performed utilising a SWH available on the local market in the city of Brisbane region, Australia. The analysed system was listed in the Register of SWHs provided by the Australian Clean Energy Regulator published on 15 December 2014 with registration valid until 31 December 2030 [11]. Tables S-A 2 and 3 detail the technical specifications of the SWH and the collectors, respectively.

Table S-A 2. Technical specifications of solar water heater for field experiment.

\begin{tabular}{ll}
\hline Parameter & SWH \\
\hline Type & Thermosiphon roof mounted \\
Tank volume (litres) & 300 \\
Circuit & Open \\
Collector type & Glazed flat-plat \\
Collector absorber coating & Polyester black paint \\
Number of collectors & 2 \\
Auxiliary electric booster power (W) & 1800 \\
\hline
\end{tabular}

Table S-A 3. Technical specifications of the thermal efficiency of collectors.

\begin{tabular}{ll}
\hline Parameter & Value \\
\hline Gross area $\left(\mathrm{m}^{2} \cdot\right.$ collector $\left.^{-1}\right)$ & 1.983 \\
Test fluid & Water \\
Test flow rate $\left(1 \cdot \mathrm{s}^{-1}\right)$ & 0.037 \\
Test correlation type & Inlet \\
Optical coefficient $($ dimensionless $)$ & 0.608 \\
Linear loss coefficient $\left(\mathrm{W} \cdot \mathrm{m}^{-2} \cdot \mathrm{K}\right)$ & -5.4707 \\
Quadratic loss coefficient $\left(\mathrm{W} \cdot \mathrm{m}^{-2} \cdot \mathrm{K}^{2}\right)$ & -0.0271
\end{tabular}




\begin{tabular}{ll}
\hline Parameter & Value \\
\hline Linear angle modifier coefficient & -0.1718 \\
Quadratic angle modifier coefficient & -0.058 \\
\hline
\end{tabular}

The field experiment system was a thermosiphon SWH fitted with two polyester black paint observer coating solar panels (Fig. S-A 1). The brand of the SWH was de-identified to preserve the anonymity of the manufacturer. The SWH was installed on a flat roof at the N44 building at Griffith University, Nathan campus in Brisbane (coordinates: $27^{\circ} 33^{\prime} 11.77^{\prime \prime}$ ' $; 153^{\circ} 03^{\prime} 07.48^{\prime \prime} \mathrm{E}$ ). The system was positioned on frames provided by a local SWHs retailer, and it was out of the influence of shades at an orientation angle of $18.0^{\circ}$ relative to true north, and a tilt angle of $17.2^{\circ}$. The experiment conditions were different from the ideal condition for Brisbane city, i.e. $0^{\circ}$ north direction and $27.5^{\circ}$ tilt [12,13]; nonetheless, the experiment may be representative of a real site condition, as the ideal roof inclination and a perfect north orientation for SWHs may be rare.

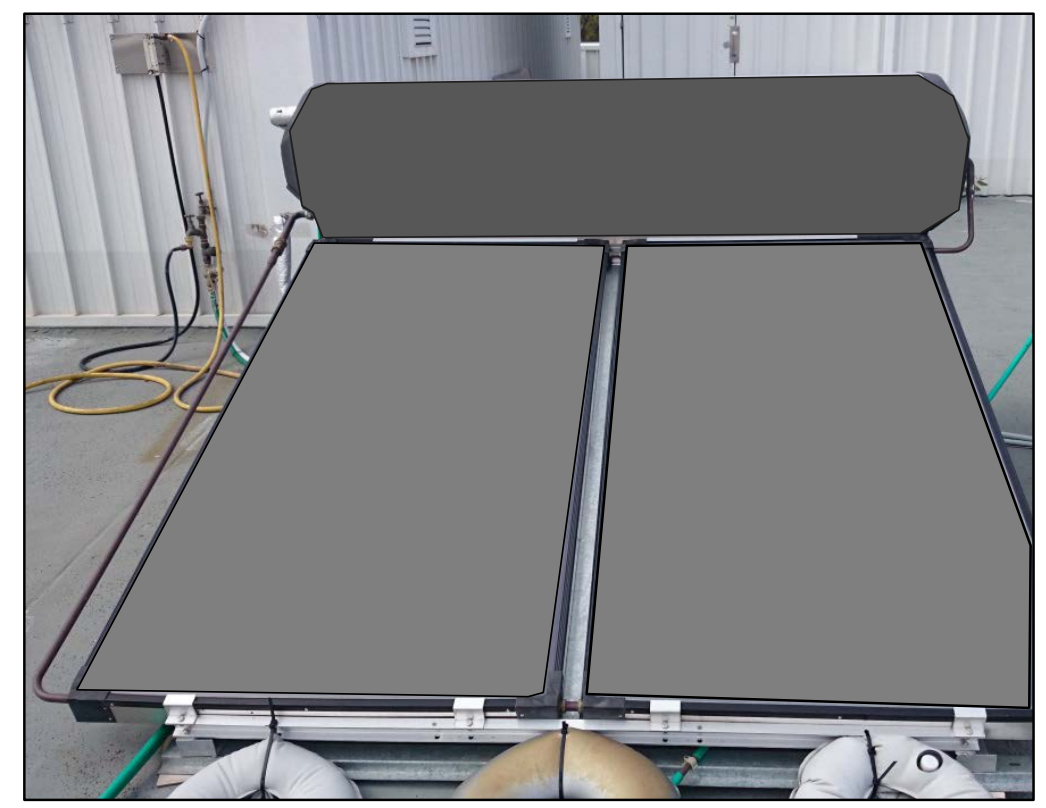

Fig. S-A 1. Field experiment with thermosiphon solar water heater.

A solenoid valve operated by a timer was used to emulate a residential hot water consumption pattern in the field experiment (Fig. S-A 2). The emulated residential hot water time of use was based on previously completed high resolution smart meter studies, i.e. Beal and Stewart [14]. The system had two operation cycles, one for weekdays and another for weekends. In the weekday cycle, water was consumed between 7:00 am and 7:30 am, 6:00 pm and 6:30 pm, and 8:00 pm and 8:15 pm; while, in the weekend cycle, consumption events occurred between 8:00 and 9:00 am, 12:00 pm and 12:30 pm, 6:00 pm and 7:00 pm. The average hot water consumption in the weekday and weekend cycles was 158 and 439 litres'day ${ }^{-1}$, respectively. The emulated water consumption pattern was intended to represent the hot water consumption in the more typical household where occupancy occurs mainly during non-business hours during weekdays and at all times on the weekends. 


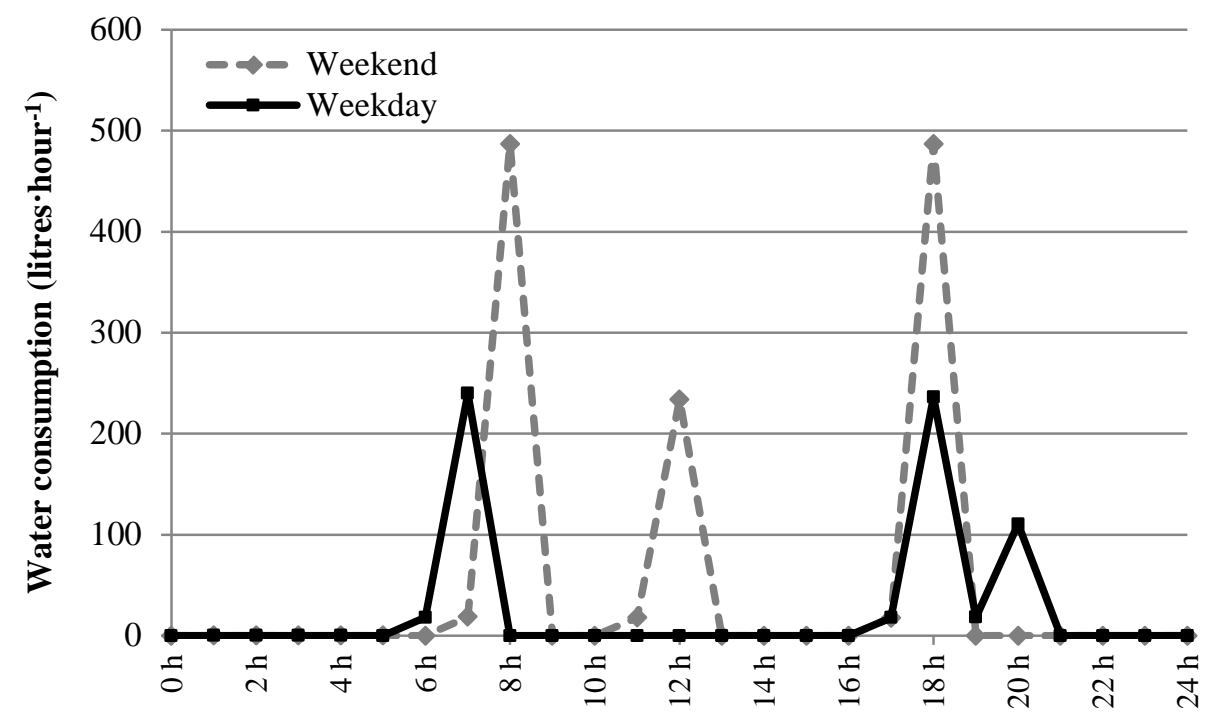

Fig. S-A 2. Total hot water consumption pattern at end-use points applied in the field experiment.

The energy and water consumption of the SWH were monitored using high resolution energy and water meters with pulse precision of $0.1 \mathrm{~W}$ and 0.014 litres, respectively. The temporal resolution of loggers was set to record pulses every five seconds. The collected water and energy consumption data was converted into diurnal demand consumption patterns with a temporal resolution of one hour by using an electronic spreadsheet.

\subsection{Comparison between theoretical and experimental results}

Computational models showed a similar trend to experimental results with power-peaks from 8 am to 9 am and $7 \mathrm{pm}$ to $9 \mathrm{pm}$. The input power-peak of the SWH auxiliary heating element reached a maximum of 1,773 W in the field experiment, which is approximately equal to the technical specification of the manufacturer (i.e. 1,800 W) utilised into the models. On average, modelled energy consumption results showed a discordance of $-0.9 \%$ in relation to experimental results, with an equivalent daily energy consumption of 12.6 and $12.8 \mathrm{kWh}$, respectively. The level of variability calculated based on the root mean square error $\left(3.1 \mathrm{kWh}^{-1 a y^{-1}}\right)$ and the average result $\left(12.6 \mathrm{kWh} \cdot \mathrm{day}^{-1}\right)$ of models was equal to $24 \%$. Fig. S-A 3 illustrates the average diurnal electricity consumption pattern from the experiment and model results.

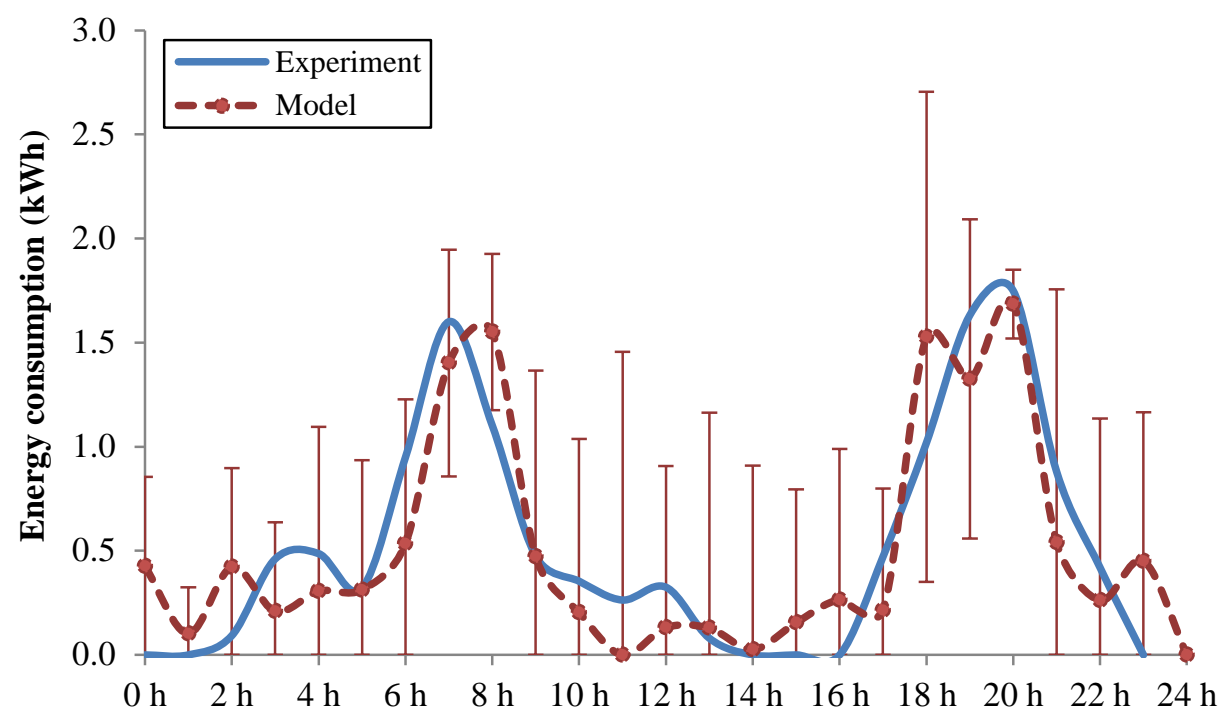


Fig. S-A 3. Average energy consumption profile of the assessed SWH from 20 September 2014 to 10 October 2014.

The error bars in Fig. S-A 3 representing the root mean square error of modelled results in relation to experimental results on an hourly basis indicates the degree of variability of modelled results. The variability of the model was less pronounced after water consumption events (e.g. 8 am and 8 pm), as such instances are usually followed by auxiliary heating events. On the other hand, electricity consumption predictions at other times had a larger variability.

Similar results are described by Griffith and Ellis [15], whom have also carried out the validation of EnergyPlus software package for solar thermal models. The authors have demonstrated that EnergyPlus and TRNSYS software packages provide the same result with minor differences only at very low solar radiance levels. Despite TRANSYS widespread use for thermal energy analysis by academics [16-19] and practitioners (e.g. AS/ANZ 4234:2008 [20]), it is a proprietary software, while EnergyPlus is a robust publicly available software. 


\section{S-B. Review of inputs parameters for sensitivity analysis}

The value range for most of the input parameters used in the sensitivity analysis was based on five energy efficiency classes, i.e. lower-extreme (LE), lower-standard (LS), standard (S), upper-standard (US) and upperextreme (UE). The variability of such values was statistically determined for stochastic parameters, whereas prior studies and expert appraisal were adopted for non-stochastic parameters range estimation. In the following sections, stochastic and non-stochastic input parameters for the sensitivity analysis are described.

\subsection{Stochastic input parameters}

Input parameters with random variability were classified as stochastic variables divided into the five energy efficiency classes described in Table S-B 1.

Table S-B 1. Stochastic variability of input parameter values for energy efficiency classes.

\begin{tabular}{|c|c|c|}
\hline $\begin{array}{l}\text { Energy } \\
\text { efficiency } \\
\text { classes }\end{array}$ & Description & $\begin{array}{l}\text { Statistical } \\
\text { correspondence }\end{array}$ \\
\hline $\begin{array}{l}\text { Upper-extreme } \\
\text { (UE) }\end{array}$ & $\begin{array}{l}\text { Upper range of values based on an extrapolation of current value } \\
\text { ranges described in the literature/technical specifications. It } \\
\text { represents extreme levels of service, site characteristics or } \\
\text { technological advancements which are not expected, common or } \\
\text { currently available, and are likely to have a beneficial impact on the } \\
\text { energy performance of systems. }\end{array}$ & $\begin{array}{l}\text { Extreme value, at a } \\
99 \% \text { confidence } \\
\text { interval, which } \\
\text { promotes an } \\
\text { enhancement in the } \\
\text { energy performance. }\end{array}$ \\
\hline $\begin{array}{l}\text { Upper-standard } \\
\text { (US) }\end{array}$ & $\begin{array}{l}\text { Upper range of values described in the literature/technical } \\
\text { specifications. It represents the maximum level of performance or the } \\
\text { most advantageous standard value currently achievable which causes } \\
\text { a beneficial impact on the energy performance of systems in } \\
\text { comparison with standard values. }\end{array}$ & $\begin{array}{l}\text { Extreme value, at a } \\
95 \% \text { confidence } \\
\text { interval, which } \\
\text { promotes an } \\
\text { enhancement in the } \\
\text { energy performance. }\end{array}$ \\
\hline Standard (S) & $\begin{array}{l}\text { Most frequent or recurrent value described in the literature/technical } \\
\text { specifications. It represents the status quo, general assumption or } \\
\text { default value applicable to a parameter. }\end{array}$ & Mean value. \\
\hline $\begin{array}{l}\text { Lower-standard } \\
\text { (LS) }\end{array}$ & $\begin{array}{l}\text { Lower range of values described in the literature/technical } \\
\text { specifications. It represents the minimum level of performance or the } \\
\text { least advantageous standard value currently required which causes a } \\
\text { detrimental impact on the energy performance of systems in } \\
\text { comparison with standard values. }\end{array}$ & $\begin{array}{l}\text { Extreme value, at a } \\
95 \% \text { confidence } \\
\text { interval, which } \\
\text { promotes a } \\
\text { reduction in the } \\
\text { energy performance. }\end{array}$ \\
\hline $\begin{array}{l}\text { Lower-extreme } \\
\text { (LE) }\end{array}$ & $\begin{array}{l}\text { Lower range of values based on an extrapolation of current value } \\
\text { ranges described in the literature/technical specifications. It } \\
\text { represents extreme levels of service, site characteristics or } \\
\text { technological limitations which are not expected, common or } \\
\text { foreseen, and are likely to have a detrimental impact on the energy } \\
\text { performance of systems. }\end{array}$ & $\begin{array}{l}\text { Extreme value, at a } \\
99 \% \text { confidence } \\
\text { interval, which } \\
\text { promotes a } \\
\text { reduction in the } \\
\text { energy performance. }\end{array}$ \\
\hline
\end{tabular}

Among the analysed stochastic inputs, five parameters were associated with the technical specification of systems, including: solar collector efficiency, solar collector area, tank volume, electric back-up heating power rate, and electric back-up heating temperature range. Moreover, stochastic input parameters were also composed of three site-specific conditions, namely: solar collector dust accumulation, cold water temperature, and hot water pipe length. The three user behaviour variables were also part of this parameter group, i.e. water 
temperature, demand and time-of-use at the end-use points. All stochastic input parameters were analysed as part of a population described by a Gaussian (normal) distribution.

The standard value class was used to represent the expected value of the distribution (i.e. mean value) for stochastic parameters. For most of the parameters, the lower-standard and the upper-standard value classes were represented by the $95 \%$ confidence interval, as this level of confidence is typically adopted into engineering projects as described elsewhere (e.g. Moffat [21], Phillips \& Eberhardt [22] and Mathioulakis et al. [23]). The broader level of confidence of $99 \%$ was utilised to describe extreme values (i.e. lower-extreme and upperextreme values), which were calculated using Eq. S-B 1 for most of the stochastic parameters.

$V_{99}=S \pm\left[\frac{z_{99}}{z_{95}} \times\left|V_{95}-S\right|\right]$

where $V_{99}$ is the extreme value at a $99 \%$ confidence interval (lower-extreme or upper-extreme values); $S$ is the mean value (standard value); $V_{95}$ is the extreme value at $95 \%$ confidence interval (lower-standard or upperstandard values); $z_{99}$ is the $99 \%$ confidence interval factor from the Gaussian distribution; and $z_{95}$ is the $95 \%$ confidence interval factor from the Gaussian distribution.

Some of the values reviewed in the literature were considered to be part of either the lower-extreme or the upper-extreme classes (99.9\% confidence interval) due to their unusual characteristics. For such parameters, the lower-standard and upper-standard classes were determined in an analogous way to the one described in Eq. S-B 1. The variability of stochastic input parameters is summarised in Table S-B 2.

Table S-B 2. Stochastic input parameters.

\begin{tabular}{|c|c|c|c|c|c|c|}
\hline \multicolumn{2}{|c|}{ Energy efficiency classes } & \multirow{2}{*}{$\begin{array}{l}\text { LE } \\
0.574 \\
(39.4 \%)\end{array}$} & \multirow{2}{*}{$\begin{array}{l}\text { LS } \\
0.608 \\
(42.7 \%)\end{array}$} & \multirow{2}{*}{$\begin{array}{l}S \\
0.691 \\
(56.2 \%)\end{array}$} & \multirow{2}{*}{$\begin{array}{l}\text { US } \\
0.784 \\
(63.5 \%)\end{array}$} & \multirow{2}{*}{$\begin{array}{l}\text { UE } \\
0.823 \\
(67.2 \%)\end{array}$} \\
\hline Solar collector & $\begin{array}{l}\text { Optical coefficient (Efficiency } \\
\%)^{*}\end{array}$ & & & & & \\
\hline & Gross area $\left(\mathrm{m}^{2}\right)^{* *}$ & 0.9 & 1.4 & 2.8 & 4.5 & 5.2 \\
\hline & $\begin{array}{l}\text { Dust effect on glass transmittance } \\
\text { reduction }(\%)\end{array}$ & 64.0 & 47.0 & 5.0 & 2.7 & 0.0 \\
\hline \multirow{4}{*}{ Storage tank } & Volume (litres) & 90 & 250 & 315 & 450 & 630 \\
\hline & Electric power rate $(\mathrm{kW})$ & 5.5 & 4.8 & 3.0 & 1.5 & 0.9 \\
\hline & Electric temperature range $\left({ }^{\circ} \mathrm{C}\right)$ & 80 & 74 & 60 & 53 & 50 \\
\hline & $\begin{array}{l}\text { Inlet temperature - cold water } \\
\left({ }^{\circ} \mathrm{C}\right)^{* * *}\end{array}$ & Avg -6 & Avg -4 & Avg & Avg +4 & $\operatorname{Avg}+6$ \\
\hline Hot water pipe & Length (metres) & 18 & 15 & 9 & 5 & 0 \\
\hline \multirow[t]{4}{*}{ End-use point } & Set-point temperature $\left(\mathrm{C}^{\circ}\right)$ & 50 & 47 & 40 & 35 & 33 \\
\hline & $\begin{array}{l}\text { Water demand (litres } \cdot \text { household } \\
{ }^{1} \cdot \text { day }^{-1} \text { ) }\end{array}$ & 233 & 210 & 155 & 100 & 77 \\
\hline & $\begin{array}{l}\text { Morning water consumption peak } \\
\text { (time)**** }\end{array}$ & $6: 50$ & $7: 00$ & $7: 30$ & 9:00 & $9: 40$ \\
\hline & Evening water consumption peak & None & $17: 00$ & 18:00 & $20: 00$ & None \\
\hline
\end{tabular}




\begin{tabular}{ccccccc}
\hline Energy efficiency classes & LE & LS & S & US & UE \\
\hline (time)**** & & & &
\end{tabular}

Note: * The upper- and lower-extreme classes of solar collectors efficiency were estimated based on the variation of the optical coefficient, a constant solar radiation of $800 \mathrm{~W} \cdot \mathrm{m}^{-2}$ and temperature delta of $20^{\circ} \mathrm{C}$ between the air and cold water inlet, and a standard solar irradiance reduction of $5 \%$ due to dust accumulation. The efficiency was used only for classification purposes, while the optical coefficient was used in models. ** Gross area of a single collector. *** Average (Avg.) difference; **** The total time of all water consumption events was equal to 20 minutes.

The efficiency of solar collectors was calculated only for flat plat solar collectors, which are the more common than other collector types (e.g. evacuated tubes) in Australia. The thermal efficiency coefficients of solar collectors were obtained from the Solar Rating and Certification Corporation of the United States (SRCC-US) [24] listed in the EnergyPlus library, including 134 glazed flat plate solar collectors. The instantaneous solar radiance was calculated using the second-order thermal efficiency equation (Eq. S-B 2) as per the AS/NZS 2535.1/2007 [25]. This equation has large application for instantaneous thermal efficiency analysis of solar collectors, e.g. Duffie and Bechman [26] among several other studies and codes.

$\eta=c_{0}+c_{1} \frac{\left(T_{i n-} T_{a i r}\right)}{I_{\text {solar }}}+c_{2} \frac{\left(T_{\text {in- }} T_{a i r}\right)^{2}}{I_{\text {solar }}}$

where $\eta$ is the thermal efficiency coefficient (dimensionless); $c_{0}$ is the optical coefficient (dimensionless); $c_{1}$ is the linear loss coefficient $\left(\mathrm{W} \cdot \mathrm{m}^{-2} \cdot \mathrm{K}\right) ; c_{2}$ is the quadratic loss coefficient $\left(\mathrm{W} \cdot \mathrm{m}^{-2} \cdot \mathrm{K}^{2}\right) ; T_{\text {in }}$ is the inlet temperature of the working fluid $\left(\mathrm{K}^{\circ}\right.$ or $\left.\mathrm{C}^{\circ}\right)$; $T_{\text {air }}$ is the outdoor air temperature $\left(\mathrm{K}^{\circ}\right.$ or $\left.\mathrm{C}^{\circ}\right)$; and $I_{\text {solar }}$ is the global incident solar radiation $\left(\mathrm{W} \cdot \mathrm{m}^{-2}\right)$.

The instantaneous thermal efficiency of collectors was utilised for the classification of solar collectors among the different energy efficiency classes, while the associated optical coefficient was employed into models. The most representative solar collector efficiency class applied in the base case scenario was the lower-standard class. Typically, the installation of SWHs in sub-tropical and tropical climates is undertaken with the use of solar collectors with efficiency levels lower than the average efficiency of collectors available on the market. The installation of solar collectors with lower efficiency levels in these regions is due to both the availability of abundant solar radiation, and cost savings. The instantaneous solar collector efficiency was calculated based on the temperature gradient prescribed by the SRCC-US for water heating systems in warm climates [24] (i.e. 20 ${ }^{\circ} \mathrm{C}$ between the inlet working fluid and the outdoor air temperatures) and an instantaneous solar radiance of 800 $\mathrm{W} \cdot \mathrm{m}^{-2}$

The gross area variability of single collectors was also analysed based on the available data from the EnergyPlus solar collector library. The total solar collector area of analysed systems was twice the size of a single collector, as models were performed for SWHs with two collectors. The minimum and the maximum gross area of collectors within the studied sample were considered as the lower- and the upper-extreme values (99\% confidence interval).

Reductions in the glass transmittance of collectors related to dust accumulation were analysed based on values reported in the literature. The dust effect assessment is a function of the tilt angle of solar collectors and the precipitation frequency in a region, as well as their associated self-cleaning capacity. For this parameter, the variation described in the literature was divided into humid and arid/semi-arid climates, where values oscillated between $2.7 \%$ [27] and 5.0\% [28] in humid climates, and from 2.5\% to 64.0\% in semi-arid or arid climates depending on the frequency of manual or mechanical cleaning events and the tilt angle of collectors [29]. Based 
on this information, the extreme values of the dust effect on collectors was assumed to be zero (upper-extreme value equal to clean collectors) and 64\% (lower-extreme value equal to the worst case scenario described in the literature [29]). The lower-extreme limit (64\%) may represent extreme events during extensive drought periods.

The variability of hot water storage tank volumes was estimated considering data from the Department of Energy and Water Supply (DEWS) as part of the Queensland Government's Open Data Initiative. Detailed information on SWHs installed as part of rebate schemes in Queensland ('Solar Hot Water Rebate' spreadsheet) was downloaded from the DEWS' website [30] and filtered considering SWHs installed in postcodes for the City of Brisbane only, i.e. 4000, 4101, 4106, 4169 and 4178. In total, 111 systems were selected, from which the minimum, median and maximum volumes of the sample were adopted as the lower-standard, standard and upper-standard categories, respectively.

The variability of the power rate of auxiliary electric water heating elements was determined through a marked review of the power rate of $20 \mathrm{SWH}$ systems. The set-point temperature of auxiliary electric water heating elements (temperature range) was defined in accordance with the temperature ranges of the thermostat used in the field experiment (i.e. Robertshaw ST13-01133). This thermostat enables the selection of a wide range of upper temperature thresholds (i.e. set-point of 50,55, 60, 65, 70, 75 and $80^{\circ} \mathrm{C}$ ) at a temperature dead band (differential) of $8{ }^{\circ} \mathrm{C}$. The minimum $\left(50^{\circ} \mathrm{C}\right)$ and maximum $\left(80^{\circ} \mathrm{C}\right)$ temperatures were considered to be representative of the lower-extreme and upper-extreme values for the temperature range of auxiliary heating elements, as these temperatures are outside the range required for Legionella spp. control and overheating protection, i.e. temperatures from 60 to $70^{\circ} \mathrm{C}$ [13].

The inlet water temperature supplied to SWHs (i.e. cold water temperature) was calculated as per the method described by Hendron et al. [31]. The two key inputs of this method, i.e. the annual average outdoor air temperature (dry-bulb) and the maximum difference in the monthly average outdoor air temperature, were calculated based on the Representative Meteorological Year (RMY) climate file for Brisbane. Variations in the cold water supply temperature related to local factors (e.g. water mains depth, water table levels, incidence of solar radiation on covering ground surface, or soil type and associated thermal capacity) were taken into consideration in accordance with oscillation ranges described by Kenway et al. [32]. The authors reported $8{ }^{\circ} \mathrm{C}$ temperature variation among different points of a water supply network within a single water supply zone in Melbourne, Australia.

The hot water pipe length between the hot water tank and the demand end-use point was estimated based on the data described by Binks et al. [33] for Melbourne and Brisbane. The authors have described the characteristics of seven hot water supply systems in residential detached dwellings. Reported parameters included the average length of hot water pipes, for which the minimum, average and maximum values were equal to 5, 9 and 18 metres, respectively. Based on these values, the upper extreme class was calculated using Eq. S-B 1, whilst the lower-extreme was considered as zero to represent a potential use of hot water from a connection point adjacent to the hot water tank.

The end-use point set-point temperature described in the literature varied between 35 and $50{ }^{\circ} \mathrm{C}$ with an average of $40{ }^{\circ} \mathrm{C}$ (e.g. [31,34,35]). The maximum end-use temperature is limited by hot water supply standards to $50^{\circ} \mathrm{C}$ [36], and hence this value was adopted as the upper-extreme temperature class. The lower-extreme temperature class $\left(33^{\circ} \mathrm{C}\right)$ was calculated based on the lower-standard value $\left(35^{\circ} \mathrm{C}\right)$ and the standard value $\left(40{ }^{\circ} \mathrm{C}\right)$ using Eq. S-B 1. The hot water consumption patterns of models were estimated taking into consideration the national per capita hot water demand and household (hh) size. This calculation was performed utilising the following information: (i) the upper and lower daily hot water demand prescribed by the Plumbing Reference Guide of Australia [13] (i.e. 50 and 70 litres $\cdot$ person $^{-1} \cdot$ day $^{-1}$ ); and (ii) the average household size in Australia (2-3 people household $\left.{ }^{-1}[37]\right)$. As a result, the total daily water consumption at hot water end-use points varied from 100 to 210 litres $\cdot$ household ${ }^{-1} \cdot$ day $^{-1}$. Such consumption was considered to be distributed within two peak-hour periods (morning and evening peaks) for standard classes as described by Vieira et al. [3], i.e. from 7:00am to 9:00am and 5:00 pm to 8:00 pm. Values for the extreme classes were calculated considering a single peak during the day based on the extrapolation from $95 \%$ to $99 \%$ confidence interval of the earliest (7:00 am) and the latest (9:00 am) hours of the morning peak described by Vieira et al. [3], resulting in a lower-extreme peak-hour at 6:50 am and an upper-extreme peak-hour at 9:40 am. 


\subsection{Non-stochastic input parameters}

The variation of non-stochastic input parameters was analysed based on data available in the literature using a similar approach to the one adopted for stochastic parameters; however, the variation of non-stochastic parameters was not described by a Gaussian distribution, but rather by assumptions for the assessed value ranges. Non-stochastic technical specification parameters encompassed: tank heat loss, and electric back-up heating position; whereas, non-stochastic site-specific parameters were formed by: solar collector direction, tilt angle, shadowing, hot water pipe insulation, and electricity tariff time-of-use. The adopted variation of nonstochastic parameters is summarised in Table S-B 3, except for input parameters based on geographical directions (i.e. solar collector direction and shadowing).

Table S-B 3. Non-stochastic input parameters.

\begin{tabular}{|c|c|c|c|c|c|}
\hline $\begin{array}{l}\text { Energy efficiency } \\
\text { classes }\end{array}$ & $\mathrm{LE}$ & LS & S & US & UE \\
\hline $\begin{array}{l}\text { Storage tank heat loss } \\
\left(\mathrm{W} \cdot \mathrm{K}^{-1}\right)\end{array}$ & 3.08 & 1.32 & 0.75 & 0.47 & 0.18 \\
\hline $\begin{array}{l}\text { Electric back-up heating } \\
\text { position (height \%)* }\end{array}$ & $0 \%$ & $25 \%$ & $50 \%$ & $75 \%$ & $100 \%$ \\
\hline Solar collector tilt angle & Vertical & Horizontal* & Half latitude & $\begin{array}{l}\text { Latitude + } \\
20^{\circ}\end{array}$ & Latitude \\
\hline $\begin{array}{l}\text { Hot water pipe } \\
\text { insulation (R-value - } \\
\mathrm{K} \cdot \mathrm{m}^{2} \cdot \mathrm{W}^{-1} \text { ) }\end{array}$ & 0 & 0.20 & 0.30 & 1.00 & Adiabatic \\
\hline $\begin{array}{l}\text { Electricity tariff time-of- } \\
\text { use (interruption } \\
\text { periods) }\end{array}$ & $\begin{array}{l}7 \mathrm{am}- \\
10 \mathrm{pm} \quad(15 \\
\text { hours })\end{array}$ & $\begin{array}{l}7 \text { am }-9 \text { am } / 1 \text { pm }-9 \\
\operatorname{pm}(10 \text { hours) }\end{array}$ & $\begin{array}{l}4 \mathrm{pm}-8 \mathrm{pm} \\
(4 \text { hours })\end{array}$ & $\begin{array}{l}5 \mathrm{pm}-8 \mathrm{pm} \\
\text { (3 hours) }\end{array}$ & $\begin{array}{l}\text { None } \\
\text { hours })\end{array}$ \\
\hline
\end{tabular}

Note: * Not applicable to thermosiphon systems.

The heat loss of hot water storage tanks was analysed considering the insulation materials described by Omer et al. [38], including the following materials and associated thermal conductivity coefficients $\left(\mathrm{W} \cdot \mathrm{m}^{-1} \cdot \mathrm{K}^{-1}\right)$ : glass fibre (0.037), polystyrene $(0.030)$, polyurethane $(0.025)$, polyisocyanurate $(0.021)$, and vacuum insulation panel (0.010). The variability of the thickness of the insulation was assumed to be equal to the one encountered in the field experiment, varying from 12 to $55 \mathrm{~mm}$. Taking into account the studied range of thermal conductivity coefficients and thicknesses for insulation materials, the heat loss coefficient and thermal resistance of the hot water tank were calculated considering one-dimensional heat transfer perpendicular to the surface using Eq. S-B 3.

$R=\frac{1}{H_{L}}=\frac{L}{k \cdot A}$

where $R$ is the thermal resistance $\left(\mathrm{K}^{\cdot} \mathrm{W}^{-1}\right) ; H_{L}$ is the heat loss coefficient $\left(\mathrm{W} \cdot \mathrm{K}^{-1}\right) ; L$ is the thickness of the insulation material $(\mathrm{m})$; $k$ is the thermal conductivity of the insulation material $\left(\mathrm{W} \cdot \mathrm{m}^{-1} \cdot \mathrm{K}^{-1}\right)$; and $A$ is the surface area considered per unit area $-1\left(\mathrm{~m}^{2}\right)$. 
The positions (height) of auxiliary water heating elements and associated thermostats were considered to vary from the bottom to the top of hot water storage tanks at five different levels equally distributed.

The solar collector tilt angle was determined for different ranges. Pursuant to the Plumber Reference Guide [13], solar collectors should be installed at local latitude angles due to the maximum annual solar radiation on solar collectors at this angle. Furthermore, the optimal solar collector tilt angles may vary $20^{\circ}$ from the latitude angle as a result of the maximisation of radiation collection during winter [39]. Also, solar collectors have been also installed at extreme angles (e.g. vertical) due to space constraints in multi-storey buildings (e.g. [40]). The other extreme position (e.g. horizontal) was also considered in the herein study. The tilt angle for the base scenario was considered to be the latitude angle (upper extreme class).

The hot water pipe insulation variation for the study was adopted considering the range described in the Plumbing Reference Guide of Australia [13]. In this guideline, the insulation R-value is reported, in which the minimum insulation level required for external locations is 0.2 for valves and 0.3 for pipes; whereas, the maximum reported R-value for pipes was 1 for Alpine climates. The R-value of 0.3 was used to represent the base scenario, as the guideline indicates this value for external pipes in Brisbane or other locations with warmer weather. The lower- and upper-standard class values were defined considering the lower- and the upper insulation levels reported for external pipes in different climates nationwide. The lower- and upper-extreme values were determined considering that external pipes can be installed either without insulation, or with a perfect insulation that inhibits any head exchange (adiabatic conditions).

Based on a market review on different supply tariffs of 13 Australian electricity distributors, the energy supply time-of-use was estimated for five tariff levels aligned with the energy efficiency classes considered in the study. In total, 24 electricity tariff schemes were evaluated, from which time-of-use information was used to estimate the levels of electricity supply interruption. The base scenario made use of the tariff without interruption, which is equivalent to all day supply tariffs and represent the lower-extreme energy efficiency class for this parameter.

The solar collector direction was evaluated consideration the positioning of solar collectors towards the north, south, east and west cardinal and inter-cardinal directions. The shade effect of adjacent building on solar collectors was also analysed considering single shading elements with dimensions of 50 metres wide and 100 metres high located at 50 metres from the middle point between the two parallel solar collectors (Fig. S-B 1). For this parameter, single shading elements were taken into account from one of the cardinal or inter-cardinal directions. The geometries of shading elements and solar collectors alike were designed using the software Google SketchUp 8.0 with the plug-in software Legacy OpenStudio 1.10.10.406 for EnergyPlus models. The base scenario (standard models) was developed considering collectors with north facing orientation without shades. 


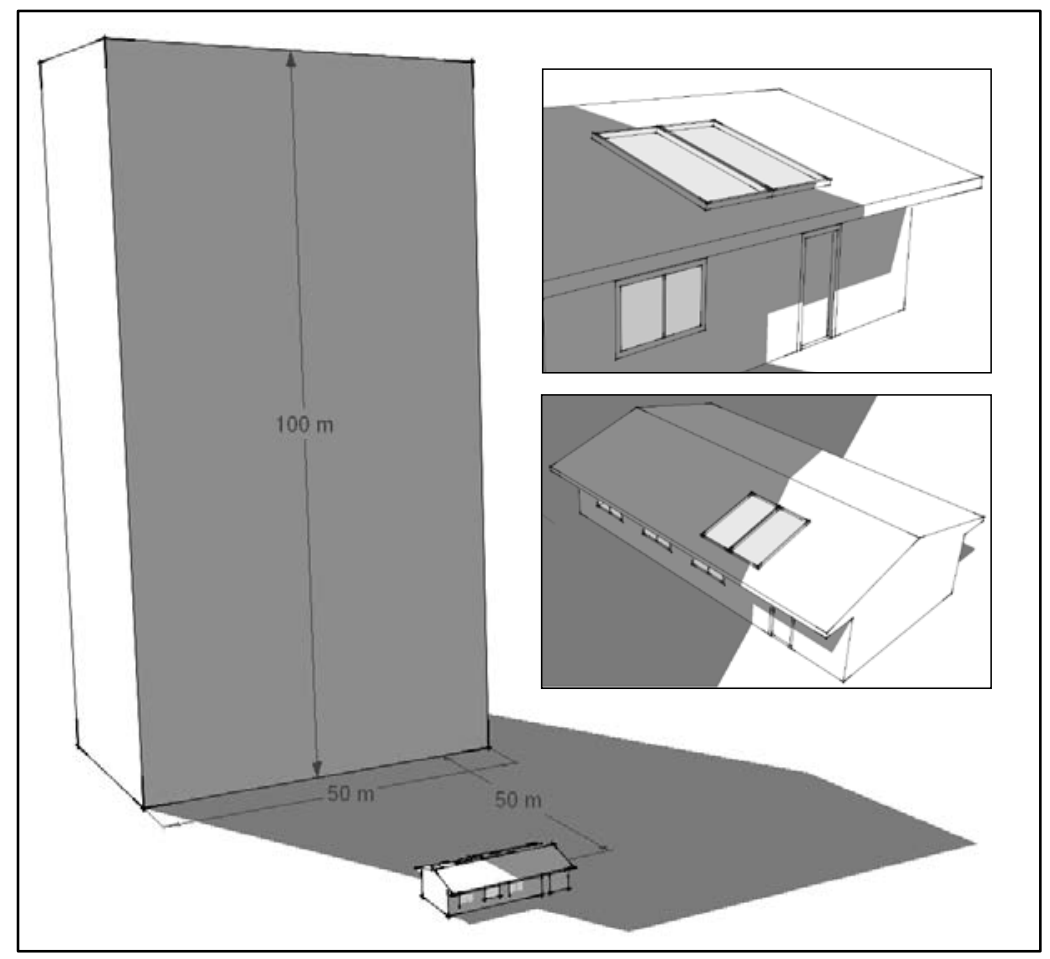

Fig. S-B 1. Representation of shadow effect on solar collectors from a single shading element positioned in the north direction.

Non-stochastic input parameters also included the storage tank position (vertical or horizontal), and the water circulation type (thermosiphon or pumping). Vertical tanks were considered to represent split systems with active pumping between solar collectors and storage tanks. For these systems, water circulation was on and off when temperatures in the collectors' outlet raised above $10^{\circ} \mathrm{C}$ and $2{ }^{\circ} \mathrm{C}$, respectively, in relation to the storage tank inlet. Horizontal tanks were designed as roof mounted thermosiphon systems with natural water circulation in solar collectors without active pumping. The hot water circulation from the collectors' outlet to the hot water tank inlet occurred when the temperature difference between the former and the latter components was $1^{\circ} \mathrm{C}$, and it stopped when the temperature difference was below $0.5^{\circ} \mathrm{C}$ as described by Kalogirou and Papamarcou [41]. The climate conditions were assessed for the sub-tropical climate of Brisbane, classified into the Bioclimatic Zone 3. For this purpose, a Representative Meteorological Years (RMY) climate file was obtained from the EnergyPlus website. 


\section{References}

[1] J. Carlo, R. Lamberts, Development of envelope efficiency labels for commercial buildings: Effect of different variables on electricity consumption, Energy Build. 40 (2008) 2002-2008, http://dx.doi.org/10.1016/j.enbuild.2008.05.002.

[2] M.M. Hassan, Y. Beliveau, Modeling of an integrated solar system, Build. Environ. 43 (2008) 804-810, http://dx.doi.org/10.1016/j.buildenv.2007.01.019.

[3] A.S. Vieira, C.D. Beal, R.A. Stewart, Residential water heaters in Brisbane, Australia: Thinking beyond technology selection to enhance energy efficiency and level of service, Energy Build. 82 (2014) 222236, http://dx.doi.org/10.1016/j.enbuild.2014.07.007.

[4] L.G. Swan, V.I. Ugursal, Modeling of end-use energy consumption in the residential sector: A review of modeling techniques, Renewable and Sustainable Energy Reviews 13 (2009) 1819-1835.

[5] Q.-Y. Li, Q. Chen, X. Zhang, Performance analysis of a rooftop wind solar hybrid heat pump system for buildings, Renew. Sustain. Energy Rev. 13 (2009) 1819-1835,

http://dx.doi.org/10.1016/j.rser.2008.09.033.

[6] D. Garcia Sanchez, B. Lacarrière, M. Musy, B. Bourges, Application of sensitivity analysis in building energy simulations: Combining first- and second-order elementary effects methods, Energy Build. 68 (2014) 741-750, http://dx.doi.org/10.1016/j.enbuild.2012.08.048.

[7] C. Lamnatou, J.D. Mondol, D. Chemisana, C. Maurer, Modelling and simulation of Building-Integrated solar thermal systems: Behaviour of the coupled building/system configuration, Renew. Sustain. Energy Rev. 48 (2015) 178-191, http://dx.doi.org/10.1016/j.rser.2015.03.075.

[8] L. Altoé, D.O. Filho, F. J. Rey, J. Carlo, Computer programs for analysis of solar domestic hot water systems: RETScreen case study Computer programs for analysis of solar domestic hot water systems : RETScreen case study, J. Energy Challenges Mech. 2015 (2015) 150-155.

[9] R.R. Perez, P. Ineichen, E.L. Maxwell, R.D. Seals, A. Zelenka, Dynamic global-to-direct irradiance conversion models, ASHRAE Trans. 98 (1992) 354-369.

[10] ASHRAE, Handbook: fundamentals, American Society of Heating, Refrigerating and Air Conditioning Engineers, New York, USA, 2013.

[11] Clean Energy Regulator, Register of solar water heaters and postcode zones, Austrailian Government (2014). http://ret.cleanenergyregulator.gov.au/Hot-Water-Systems/Eligible-Solar-WaterHeaters/eligible-swhs (Accessed 01/Feb/2017)

[12] DRET, Solar Water Heater Guide for Households, Department of Resources, Energy and tourism, Canberra, Australia, 2013. ISBN 978-1-922106-93-3 (Online).

[13] DCCEF, Plumber Reference Guide - Solar \& Heat Pump Hot Water Systems, Department of Climate Change and Energy Efficiency - Commonwealth of Australia, 2010.

[14] C. Beal, R.A. Stewart, South East Queensland Residential End Use Study: Final Report. Urban Water Security Research Alliance Technical Report No . 47, Gold Coast, Australia, 2011. ISSN 1836-5566 (Online).

[15] B.T. Griffith, P.G. Ellis, Photovoltaic and Solar Thermal Modeling with the EnergyPlus Calculation Engine, in: World Renewable Energy Congress VIII and Expo, Denver, Colorado, August 29 to September 3, 2004: pp. 1-5., http://www.nrel.gov/docs/fy04osti/36275.pdf (Accessed 01/Feb/2017)

[16] L.M. Ayompe, A. Duffy, S.J. McCormack, M. Conlon, Validated TRNSYS model for forced circulation solar water heating systems with flat plate and heat pipe evacuated tube collectors, Appl. Therm. Eng. 31 (2011) 1536-1542, http://dx.doi.org/10.1016/j.applthermaleng.2011.01.046. 
[17] A. Hobbi, K. Siddiqui, Optimal design of a forced circulation solar water heating system for a residential unit in cold climate using TRNSYS, Sol. Energy 83 (2009) 700-714, http://dx.doi.org/10.1016/j.solener.2008.10.018.

[18] J.B.A. Lima, R.T.A. Prado, V.M. Taborianski, Optimization of tank and flat-plate collector of solar water heating system for single-family households to assure economic efficiency through the TRNSYS program, Renew. Energy. 31 (2006) 1581-1595, http://dx.doi.org/10.1016/j.renene.2005.09.006.

[19] U. Jordan, K. Vajen, Influence of the DHW load profile on the fractional energy savings: a case study of a solar combi-system with TRNSYS simulations, Sol. Energy 69 (2001) 197-208.

[20] AS/NZS 4234:2008, Heated water systems - Calculation of energy consumption, 2008.

[21] R.J. Moffat, Contributions to the Theory of Single-Sample Uncertainty Analysis, Trans. Am. Soc. Mech. Eng. 104 (1982) 1-9.

[22] S.D. Phillips, K.R. Eberhardt, B. Parry, Guidelines for expressing the uncertainty of measurement results containing uncorrected bias, J. Res. Natl. Inst. Stand. Technol. 102 (1997) 577-585, http://dx.doi.org/10.6028/jres.102.039.

[23] E. Mathioulakis, G. Panaras, V. Belessiotis, Uncertainty in estimating the performance of solar thermal systems, Sol. Energy. 86 (2012) 3450-3459, http://dx.doi.org/10.1016/j.solener.2012.07.025.

[24] SRCC, Directory of SRCC certified solar collertos ratings, Solar Rating and Certication Corporation, Florida, USA, 2007.

[25] AS/NZS 2535.1:2007, Test methods for solar collectors - Part 1: Thermal performance of glazed liquid heating collectiors including pressure drop (ISO 9806-1:1994, MOD), 2007.

[26] J.A. Duffie, W.A. Beckman, Solar Engineering of thermal Processes, Wiley-Intescience, New York, 1991.

[27] H.C. Hottel, B.B. Woertz, The performance of flat-plate solar -heat collectors, Am. Soc. Mech. Eng. Trans. 64 (1942) 91-104.

[28] A.G.H. Dietz, Introduction to the utilization of solar energy, in: A.M. Zarem, D.D. Erway (Eds.), Diathermassous Mater. Prop. Mater., McGraw-Hill, New York, 1963.

[29] A.A. Hegazy, Effect of dust accumulation on solar transmittance through glass covers of plate-type collectors, Renew. Energy 2001 (2001) 525-540.

[30] DEWS, Open Data, Energy: Solar Hot Water Rebate, Department of Energy and Water Supply, Queensland Government, Australia, 2016. https://www.dews.qld.gov.au/our-department/data (Accessed 10/Feb/2017)

[31] R. Hendron, R. Anderson, C. Christensen, M. Eastment, P. Reeves, Development of an Energy Savings Benchmark for All Residential End-Uses, in: SIMBUILD2004 Conference, Boulder, Colorado, August 4-6, 2004: pp. 1-11.

[32] S. Kenway, A. Blinks, J. Bors, F. Pamminger, P. Lant, B. Head, T. Taimre, A. Grace, J. Fawcett, S. Johnson, J. Yeung, R. Scheidegger, H.-P Bader, Understanding and managing water-related energy use in Australian households, Water 41 (2014) 184-188.

[33] A.N. Binks, S.J. Kenway, P.A. Lant, B.W. Head, Understanding Australian household water-related energy use and identifying physical and human characteristics of major end uses, J. Clean. Prod. 135 (2016) 892-906, http://dx.doi.org/10.1016/j.jclepro.2016.06.091.

[34] I.R. Pillai, R. Banerjee, Methodology for estimation of potential for solar water heating in a target area, Sol. Energy 81 (2007) 162-172, http://dx.doi.org/10.1016/j.solener.2006.04.009.

[35] X. Li, W. Wu, X. Zhang, W. Shi, B. Wang, Energy saving potential of low temperature hot water system based on air source absorption heat pump, Appl. Therm. Eng. 48 (2012) 317-324, http://dx.doi.org/10.1016/j.applthermaleng.2011.12.045. 
[36] AS 3498-2009, Authorization requirements for plumbing product-Water heaters and hot-water storage tanks, Australian Standard, 2009.

[37] ABS, Year Book Australia, 2009-2010: Households and Families, Austalian Bureau of Statatistics, Austrlian Government, 2010.

http://www.abs.gov.au/AUSSTATS/abs@.nsf/lookup/916F96F929978825CA25773700169C65?opendo cument (Accessed 01/Feb/2017)

[38] S.A. Omer, S.B. Riffat, G. Qiu, Thermal insulations for hot water cylinders : a review and a conceptual evaluation, Build. Serv. Eng. Res. Technol. 28 (2007) 275-293.

[39] A. Shariah, M.A. Al-Akhras, I.A. Al-Omari, Optimizing the tilt angle of solar collectors, Renew. Energy. 26 (2002) 587-598, http://dx.doi.org/10.1016/S0960-1481(01)00106-9.

[40] A. K. Furundzic, V. Kosoric, K. Golic, Potential for reduction of CO2 emissions by integration of solar water heating systems on student dormitories through building refurbishment, Sustain. Cities Soc. 2 (2012) 50-62, http://dx.doi.org/10.1016/j.scs.2011.10.005.

[41] S.A. Kalogirou, C. Papamarcou, Modelling of a thermosyphon solar water heating system and simple model validation, Renew. Energy 21 (2000) 471-493. 